\title{
Coupled boundary-element scheme for eddy-current computation
}

\section{Journal Article}

\section{Author(s):}

Hiptmair, Ralf; Ostrowski, Jörg

Publication date:

2005-03

Permanent link:

https://doi.org/10.3929/ethz-b-000025268

\section{Rights / license:}

In Copyright - Non-Commercial Use Permitted

\section{Originally published in:}

Journal of Engineering Mathematics 51(3), https://doi.org/10.1007/s10665-004-2116-3 


\title{
Coupled boundary-element scheme for eddy-current computation
}

\author{
RALF HIPTMAIR $^{1}$ and JÖRG OSTROWSKI ${ }^{1}$ \\ Seminar for applied Mathematics, ETH Zürich, CH-8092, Zürich, Switzerland (hiptmair@sam.math.ethz.ch); ${ }^{1} A B B$ \\ Corporate Research, Baden-Dättuil, Switzerland (joerg.ostrowski@ch.abb.com)
}

Received 21 October 2003; accepted in revised form 2 August 2004

\begin{abstract}
The mathematical foundation of a symmetric boundary-element method for the computation of eddy currents in a linear homogeneous conductor which is exposed to an alternating magnetic field is presented. Starting from the A-based variational formulation of the eddy-current equations and a related transmission problem, the problem inside and outside the conductors is reformulated in terms of integral equations on the boundary of the conductors. Surface currents occur as new unknowns of this direct formulation. The integral equations can be coupled in a symmetric fashion using the transmission conditions for the vector potential $\mathbf{A}$ and the magnetic field $\mathbf{H}$. The resulting variational problem is elliptic in suitable trace spaces. A conforming Galerkin boundary-element discretization is employed, which relies on surface edge elements and provides quasi-optimal discrete approximations for the tangential traces of $\mathbf{A}$ and $\mathbf{H}$. Surface stream functions supplemented with co-homology vector fields ensure the vital zero divergence of the discrete equivalent surface currents. Simple expressions allow the computation of approximate total Ohmic losses and surface forces from the discrete boundary data.
\end{abstract}

Key words: boundary elements, divergence-conforming boundary elements, eddy-current problem, surface stream functions, homology spaces

\section{Introduction}

The typical setting of eddy-current problems involves a bounded conducting region $\Omega_{C}$ and its complement $\Omega^{\prime}:=\mathbb{R}^{3} \backslash \bar{\Omega}_{C}$, the non-conducting homogeneous, isotropic air region. Usually, $\Omega^{\prime}$ is supposed to have the electromagnetic properties of empty space $\left(\boldsymbol{\epsilon}=\epsilon_{0}, \boldsymbol{\mu}=\mu_{0}\right)$, whereas $\Omega_{C}$ might be filled with some "complex" conducting material. In this paper we restrict our attention to the case of a simple, linear, homogeneous, and isotropic conductor characterized by a constant conductivity $\sigma>0$ and permeability $\mu_{c}>0$. This can be a reasonable approximation for a non-ferromagnetic material like aluminum.

In eddy-current simulation the shape of the conductor is usually provided in some CAD format. Therefore, we can take for granted that the surface of $\Omega_{C}$ is piecewise smooth and consists of a few curved faces. All the developments of this paper refer to such a geometric setting.

Electromagnetic excitation will be provided by an ideal coil located off $\Omega_{C}$, and it can be modeled by a compactly supported current density $\mathbf{j}_{s}=\mathbf{j}_{s}(\mathbf{x}, t)$, for which we demand $\operatorname{div} \mathbf{j}_{s}=$ 0 . We will only examine the case of a time-harmonic excitation with fixed angular frequency $\omega>0$. Thanks to the linearity of all materials involved, this will permit us to switch to the frequency domain and state the equations in terms of complex amplitudes (phasors) of all electrodynamic quantities.

The goal of the numerical simulation may be the approximate computation of the total Ohmic losses in the conductors, and of the electromagnetic forces acting on the conductor. This entails discretizing the field equations and, in particular, coping with the unbounded part $\Omega^{\prime}$ of the generic computational domain $\mathbb{R}^{3}$. The standard approach is the finite-element 
method, in which artificial homogeneous boundary conditions for the fields are imposed "sufficiently" far away from the conductor. This is justified by the decay properties of the fields, although it may be difficult to fix a viable cut-off distance a priori.

After meshing the resulting bounded computational domain, the finite-element discretization can proceed in the standard fashion. However, in case of a complicated geometry of $\Omega_{C}$ suitable finite-element meshes may contain a prohibitively large number of elements.

Boundary-element methods (BEM) applied to the field equations in $\Omega^{\prime}$ steer clear of all these difficulties, since they are based on integral equations on the surface $\partial \Omega_{C}$ that employ an explicit fundamental solution of the partial differential equation satisfied by a field. Such a reduction to equations on $\partial \Omega$ is only feasible for homogeneous equations with constant coefficients, and this is just the setting we take for granted. The boundary-integral equations can be recast in weak form, which yields variational equations posed on Hilbert spaces of traces. The final discrete equations can be obtained by a straightforward Galerkin discretization. Finite elements built upon a triangulation of $\partial \Omega_{C}$ can be used for this purpose. These are commonly known as boundary elements.

The boundary-element method offers a very attractive option in computational electromagnetism [1-5] and it also plays an important role in eddy-current computation [6-11]. BEMs come in many varieties: we can distinguish direct and indirect boundary-integral equations, which can be discretized by means of the Nystrøm method, collocation or Galerkin techniques. In this article, the focus will be on the Galerkin approach.

The boundary-integral equations of the direct method link the Cauchy data of the problem. In an electromagnetic context, the Cauchy data are the tangential components of electric and magnetic fields. Hence, the natural transmission conditions for electromagnetic fields immediately lend themselves to the coupling of boundary-integral equations for $\Omega_{C}$ and $\Omega^{\prime}$. A judicious coupling strategy introduced by M. Costabel [12] relies on the so-called Calderón projectors and leads to a symmetric structure of the coupled problem. The present article will adapt this method to the eddy-current problem to derive a linear system of equations whose unknowns are associated with the edges and vertices of a surface mesh on $\partial \Omega_{C}$.

If the conductor is non-homogeneous or is made of a ferro-magnetic material (steel), the boundary-element method is no longer available for the treatment of the interior eddy-current problem in $\Omega_{C}$, and a finite-element discretization based on curl-conforming edge elements on a volume mesh is required $[13,14]$. However, the problem in $\Omega^{\prime}$ is still amenable to boundaryelement methods, which can be coupled with the finite-element equations using ideas similar to those discussed in this paper; see [15].

However, the skin effect will lead to sharp boundary layers of the fields inside $\Omega_{C}$, which enforces the use of rather fine aligned finite-element meshes close to $\partial \Omega_{C}$. The boundary-element method, however, has the boundary layers built into its representation formulas and is not affected. This is another reason for using BEM whenever possible.

In fact, the skin effect can be harnessed for numerical approximation by replacing the eddy-current equations inside the conductor with so-called impedance boundary conditions [2, Section 4.7]. These provide an acceptable approximation, if we deal with good conductors and if $\partial \Omega_{C}$ is smooth. We will elaborate on how impedance boundary conditions can be coupled with BEM for the eddy-current problem in $\Omega^{\prime}$.

\section{The eddy-current model}

The behavior of an electromagnetic field is governed by Maxwell's equations. Instead of using these, in special situations simplified quasistatic models supply sufficiently accurate 
approximations to the true fields [16]. One of them is the eddy-current model, representing a magneto-quasistatic approximation to Maxwell's equations in the sense that the electric field energy is neglected. This model is reasonably accurate for slowly varying fields, for which the change in magnetic field energy is dominant $[16,17]$. "Slowly varying", means that

$$
L \sqrt{\epsilon \mu} \omega \ll 1,
$$

where $L$ is the characteristic size of the region of interest: $\Omega_{C}$ has to be small compared to the wavelength of electromagnetic waves, which makes it possible to ignore wave propagation. There is a second condition for the validity of the eddy-current approximation, requiring that the typical time-scale is long compared to the relaxation time for space charges, that is, the conductivity must be large enough so that

$$
\omega \frac{\epsilon}{\sigma} \ll 1 .
$$

This implies that no space charges need to be taken into account. We point out that (1) and (2) provide a "rule of thumb", but ignore the impact of geometry: in the presence of thin conducting sheets or pointed tips the eddy-current approximation might become invalid locally [18, Chapter 8].

Formally, the eddy-current model arises from Maxwell's equations by dropping the displacement current $\mathbf{D}$. In the frequency domain the eddy-current model for scaled non-dimensional complex field amplitudes (for the electric field $\mathbf{E}$ and the magnetic field $\mathbf{H}$ ) reads

$$
\operatorname{curl} \mathbf{E}=-\mathbf{i} \omega \mu \mathbf{H} \quad \text { in } \mathbb{R}^{3}, \quad \operatorname{curl} \mathbf{H}=\left\{\begin{array}{ll}
\sigma \mathbf{E} & \text { in } \Omega_{C} \\
\mathbf{j}_{s} & \text { in } \Omega^{\prime}
\end{array} .\right.
$$

According to the aforementioned assumptions, the permeability $\mu$ is constant $\equiv \mu_{c}$ on $\Omega_{C}$ and equal to $\mu_{0}$ in the air region $\Omega^{\prime}$. The conductivity $\sigma$ is constant in $\Omega_{C}$ and set to zero in $\Omega^{\prime}$. The first equation is called Faraday's law, the second (reduced) Ampere's law. These equations have to be supplemented by the decay conditions

$$
\mathbf{H}(\mathbf{x})=O\left(|\mathbf{x}|^{-1}\right), \quad \mathbf{E}(\mathbf{x})=O\left(|\mathbf{x}|^{-1}\right) \quad \text { uniformly for }|\mathbf{x}| \rightarrow \infty .
$$

The so-called A-based formulation departs from (3) and eliminates $\mathbf{H}$, which results in

$$
\operatorname{curl} \mu^{-1} \operatorname{curl} \mathbf{A}+\mathrm{i} \omega \sigma \mathbf{A}=-\mathbf{j}_{s} \quad \text { in } \mathbb{R}^{3},
$$

In fact, $\mathbf{A}=(\mathrm{i} \omega)^{-1} \mathbf{E}$ is a vector potential subject to the so-called temporal gauge that makes the scalar potential vanish.

Obviously, we cannot expect a solution for $\mathbf{A}$ to be unique, because it can be altered by any gradient supported in $\Omega^{\prime}$ and will still satisfy the equations. The solution for $\mathbf{H}$ will not be affected. This reflects the fact that in a magnetoquasistatic model, $\mathbf{E}$ is relegated to the role of a "fictitious quantity". Imposing the constraints

$$
\operatorname{div} \mathbf{A}=0 \quad \text { in } \Omega^{\prime} \text { and } \int_{\Gamma_{k}} \mathbf{A} \cdot \mathbf{n} \mathrm{d} S=0,
$$

where $\Gamma_{k}, k=1, \ldots, L$, are the connected components of $\Gamma$, will restore uniqueness of a solution for $\mathbf{A}$. This is a typical gauging procedure that does not affect the physically meaningful quantities.

Next, we aim to formulate (5) as a transmission problem. To this end, we search for the right trace operators for electromagnetic fields. By a trace operator, we mean a linear mapping that extracts suitable boundary values from a field. It is well known that the relevant 


\section{R. Hiptmair and J. Ostrowski}

traces for electromagnetic fields return the tangential components of vector fields. Since we have eliminated the magnetic field in the A-based formulation (5), we should consider the following trace operators for a (smooth) vector field $\mathbf{U}$ :

Electric trace: $\quad \gamma_{D} \mathbf{U}(\mathbf{x}):=(\mathbf{n}(\mathbf{x}) \times \mathbf{U}(\mathbf{x})) \times \mathbf{n}(\mathbf{x}), \quad \mathbf{x} \in \Gamma$,

Magnetic trace: $\quad \gamma_{N} \mathbf{U}(\mathbf{x}):=\operatorname{curl} \mathbf{U}(\mathbf{x}) \times \mathbf{n}(\mathbf{x}), \quad \mathbf{x} \in \Gamma$.

Here, $\mathbf{n}$ stands for the exterior unit normal vector field on $\Gamma:=\partial \Omega_{C}$ pointing from $\Omega_{C}$ into $\Omega^{\prime}$. With technical geometries in mind, we can always assume that $\Omega_{C}$ has a piecewise smooth Lipschitz surface so that $\mathbf{n}$ will be defined almost everywhere on $\partial \Omega_{C}$. We follow the convention that exterior traces will be labeled by a superscript + , whereas traces from $\Omega_{C}$ bear a superscript - .

The trace operators are key to stating transmission conditions for the eddy-current problem across $\Gamma:=\partial \Omega_{C}$ : we require that on $\Gamma$

$$
\gamma_{D}^{+} \mathbf{A}-\gamma_{D}^{-} \mathbf{A}=0 \text { and } \frac{1}{\mu_{c}} \gamma_{N}^{-} \mathbf{A}-\frac{1}{\mu_{0}} \gamma_{N}^{+} \mathbf{A}\left(=[\mathbf{H} \times \mathbf{n}]_{\Gamma}\right)=0 .
$$

In order to state the transmission problem for the case of an excitation by $\mathbf{j}_{s}$, a corresponding "offset field" can be introduced by the Newton potential

$$
\mathbf{A}_{s}(\mathbf{x})=\frac{\mu_{0}}{4 \pi} \int_{\mathbb{R}^{3}} \frac{\mathbf{j}_{s}(\mathbf{y})}{|\mathbf{x}-\mathbf{y}|} \mathrm{d} \mathbf{y},
$$

which satisfies the gauged eddy-current equations in $\Omega^{\prime}$. For simple current distributions, it is really feasible to get closed form expressions for $\mathbf{A}_{s}$. For general $\mathbf{j}_{s}$, Equation (8) has to be evaluated numerically. This can be done with moderate computational effort, because usually $\mathbf{j}_{s}$ has a small support, and $\mathbf{A}_{s}$ is only needed on $\Gamma$.

Now we are in a position to restate the eddy-current problem in the form of a transmission problem using the total field $\mathbf{A}$ inside $\Omega_{C}$ and the reaction field $\mathbf{U}:=\mathbf{A}-\mathbf{A}_{s}$ in $\Omega^{\prime}$.

$$
\begin{aligned}
& \text { curl curl } \mathbf{A}+\mathrm{i} \omega \mu_{c} \sigma \mathbf{A}=0 \quad \text { in } \Omega_{C}, \\
& \gamma_{D}^{+} \mathbf{U}-\gamma_{D}^{-} \mathbf{A}=-\gamma_{D}^{+} \mathbf{A}_{s} \\
& \frac{1}{\mu_{0}} \gamma_{N}^{+} \mathbf{U}-\frac{1}{\mu_{c}} \gamma_{N}^{-} \mathbf{A}=-\frac{1}{\mu_{0}} \gamma_{N}^{+} \mathbf{A}_{s} \\
& \text { curl curl } \mathbf{U}=0 \quad \text { and } \quad \operatorname{div} \mathbf{U}=0 \quad \text { in } \Omega^{\prime} .
\end{aligned}
$$

which has to be supplemented by the constraints $\int_{\Gamma_{k}} \mathbf{U} \cdot \mathbf{n} \mathrm{d} S=0$, see (6), and the decay conditions (4) for $\mathbf{U}$. Existence and uniqueness of weak solutions of the transmission problem can be established [15, Theorem 2.1], [19].

If we decide to model the interaction of the conductors with the electromagnetic fields by means of impedance boundary conditions, the transmission problem can be converted into an exterior boundary-value problem. This is straightforward, because in this case we assume that the interior Cauchy data are connected by the local relationship

$$
\gamma_{D}^{-} \mathbf{A}=\eta \gamma_{N}^{-} \mathbf{A}
$$

with surface impedance $\eta:=(1+\mathrm{i}) \sqrt{\omega \mu_{c} / 2 \sigma}$. This formula is often applied even for non-constant $\sigma$ and $\mu_{c}$. In any case, $\eta$ will be a bounded function with strictly positive real part. 


\section{Boundary-integral equations}

It is obvious from (9) and (11) that the partial differential equations in $\Omega_{C}$ and $\Omega^{\prime}$ have distinct features. Consequently, we will have to use different boundary-integral representations. The crucial building blocks of such representation formulas are potentials, which are linear operators mapping data on the boundary $\Gamma$ to smooth functions defined in $\Omega_{C} \cup \Omega^{\prime}$. Here, these potentials are given by convolution operators with the kernel [20, Chapter 9]

$$
G_{\kappa}(\mathbf{x}, \mathbf{y}):=\frac{\exp (-\kappa|\mathbf{x}-\mathbf{y}|)}{4 \pi|\mathbf{x}-\mathbf{y}|}, \quad \mathbf{x} \neq \mathbf{y}
$$

for $\kappa \in \mathbb{C}$. The kernel is used to define the scalar single-layer potential

$$
\Psi_{V}^{\kappa}(\varphi)(\mathbf{x})=\int_{\Gamma} G_{\kappa}(\mathbf{x}, \mathbf{y}) \varphi(\mathbf{y}) \mathrm{d} S(\mathbf{y}), \quad \mathbf{x} \notin \Gamma,
$$

the vectorial single-layer potential

$$
\Psi_{A}^{\kappa}(\lambda)(\mathbf{x}):=\int_{\Gamma} G_{\kappa}(\mathbf{x}, \mathbf{y}) \boldsymbol{\lambda}(\mathbf{y}) \mathrm{d} S(\mathbf{y}) \quad \mathbf{x} \notin \Gamma,
$$

and the "Maxwell double-layer potential".

$$
\Psi_{M}^{\kappa}(\mathbf{v}):=\operatorname{curl} \Psi_{A}^{\kappa}(\mathbf{v} \times \mathbf{n}) .
$$

Recall that the kernal $G_{\kappa}(\mathbf{x}, \mathbf{y})$ is the fundamental solution of the differential operator $-\Delta+\kappa^{2}$ in three space dimensions. This implies, away from $\Gamma$ in a pointwise sense, $(-\Delta+$ $\left.\kappa^{2}\right) \Psi_{V}^{\kappa}(\varphi)=0$ and $\left(-\Delta+\kappa^{2}\right) \Psi_{A}^{\kappa}(\varphi)=0$. Using this in conjunction with the pointwise identity curl curl $-\operatorname{grad} \operatorname{div}=-\boldsymbol{\Delta}$, we find for the Maxwell double-layer potential,

$$
\text { curl curl } \Psi_{M}^{\kappa}(\mathbf{v})=-\Delta \operatorname{curl} \Psi_{A}^{\kappa}(\mathbf{v} \times \mathbf{n})=-\kappa^{2} \Psi_{M}^{\kappa}(\mathbf{v}),
$$

pointwise in $\mathbb{R}^{3} \backslash \Gamma$. In order to establish a similar formula for the vectorial single-layer potential, we use the identity [21, Lemma 2.3]

$$
\operatorname{div} \Psi_{A}^{\kappa}(\lambda)=\Psi_{V}^{\kappa}\left(\operatorname{div}_{\Gamma} \lambda\right) \quad \text { pointwise in } \mathbb{R}^{3} \backslash \Gamma .
$$

Here, $\operatorname{div}_{\Gamma}$ designates the surface divergence of a tangential vector field. On the smooth faces of $\Gamma$, it can be defined using local parameterizations [3, Section 2.5.2]. The same is true of the surface gradient $\operatorname{grad}_{\Gamma}$ and the surface rotation $\operatorname{curl}_{\Gamma}$. The latter satisfies $\operatorname{grad}_{\Gamma} \varphi=\operatorname{curl}_{\Gamma} \varphi \times \mathbf{n}$.

The identity (16) holds for tangential vector fields $\lambda$ for which $\operatorname{div}_{\Gamma} \lambda$ "makes sense" (more precisely, $\lambda \in \mathbf{X}_{\text {mag }}(\Gamma)$, see the explanations about trace spaces below). Thus, we can conclude

$$
\text { curl curl } \begin{aligned}
\Psi_{A}^{\kappa}(\lambda) & =(-\Delta+\operatorname{grad} \operatorname{div}) \Psi_{A}^{\kappa}(\lambda) \\
& =-\kappa^{2} \Psi_{A}^{\kappa}(\lambda)+\operatorname{grad} \Psi_{V}^{\kappa}\left(\operatorname{div}_{\Gamma} \lambda\right) .
\end{aligned}
$$

An implication of (15) and (17) is summarized in the following lemma.

Lemma 3.1. For sufficiently smooth (in the sense of trace spaces $\mathbf{X}_{\mathrm{el}}(\Gamma)$ and $\mathbf{X}_{\mathrm{mag}}(\Gamma)$, see below) tangential vector fields $\mathbf{v}$ and $\lambda$ on $\Gamma$ and $\kappa \neq 0$, the potentials

$$
\mathbf{U}=\Psi_{A}^{\kappa}(\boldsymbol{\lambda})-\frac{1}{\kappa^{2}} \operatorname{grad} \Psi_{V}^{\kappa}\left(\operatorname{div}_{\Gamma} \lambda\right) \text { and } \mathbf{V}=\Psi_{M}^{\kappa}(\mathbf{v})
$$

are homogeneous solutions of curl curl $+\kappa^{2}$ in $\mathbb{R}^{3} \backslash \Gamma$. 
Potentials are important, because they are building blocks for the following representation formulas that are closely related to the Stratton-Chu formula [22, Theorem 6.1], [21, Section 2], and [3, Section 5.5].

Theorem 3.2. If a field $\mathbf{A}: \Omega_{C} \mapsto \mathbb{C}^{3}$ solves (9), then it has a representation

$$
\begin{aligned}
& \mathbf{A}(\mathbf{x})=\Psi_{A}^{\kappa}\left(\gamma_{N}^{-} \mathbf{A}\right)(\mathbf{x})+\Psi_{M}^{\kappa}\left(\gamma_{D}^{-} \mathbf{A}\right)(\mathbf{x})-\frac{1}{\kappa^{2}} \operatorname{grad} \Psi_{V}^{\kappa}\left(\operatorname{div}_{\Gamma}\left(\gamma_{N}^{-} \mathbf{A}\right)\right)(\mathbf{x}), \\
& \mathbf{x} \in \Omega_{C}, \text { where } \kappa:=\frac{1}{2}(1+\mathrm{i}) \sqrt{2 \mu_{c} \sigma \omega}, \text { that is, } \kappa^{2}=\mathrm{i} \mu_{c} \sigma \omega .
\end{aligned}
$$

The representation formula in $\Omega^{\prime}$ also requires a trace that fits the div-operator. It is the "flux trace" $\left(\gamma_{\mathbf{n}} \mathbf{U}\right)(\mathbf{x}):=\mathbf{U}(\mathbf{x}) \cdot \mathbf{n}(\mathbf{x}), \mathbf{x} \in \Gamma$, that extracts the normal component of a vector field.

Theorem 3.3. If a vector field $\mathbf{U}: \Omega^{\prime} \mapsto \mathbb{C}^{3}$ solves (10) and satisfies the decay conditions $\mathbf{U}(\mathbf{x})=$ $O(1 /|\mathbf{x}|)$ uniformly for $|\mathbf{x}| \rightarrow \infty$, then it possesses the representation

$$
\mathbf{U}(\mathbf{x})=-\Psi_{A}^{0}\left(\gamma_{N}^{+} \mathbf{U}\right)-\Psi_{M}^{0}\left(\gamma_{D}^{+} \mathbf{U}\right)-\operatorname{grad} \Psi_{V}^{0}\left(\gamma_{\mathbf{n}}^{+} \mathbf{U}\right), \quad \mathbf{x} \in \Omega^{\prime} .
$$

We point out that the representation formulas hold in a pointwise sense off the boundary $\Gamma$ and up to $\Gamma$ in the sense of distributions.

Remark 3.1. The presence of normal boundary data in the representation formula of Theorem 3.3 constitutes a stark difference of the two representation formulas. This can be attributed to the divergence gauge necessary in $\Omega^{\prime}$. In a sense, we are dealing with a regularized problem in $\Omega^{\prime}$, for which the underlying differential operator is essentially different from that in $\Omega_{C}$.

Boundary-integral equations are obtained by applying trace operators to representation formulas. There is a wealth of choices corresponding to different combinations of traces with potentials. For $\kappa \in \mathbb{C}$ we define the following operators,

$$
\begin{aligned}
\mathbf{V}^{\kappa}:=\frac{1}{2}\left(\gamma^{-}+\gamma^{+}\right) \Psi_{V}^{\kappa}, & \mathbf{B}^{\kappa}:=\frac{1}{2}\left(\gamma_{N}^{-}+\gamma_{N}^{+}\right) \boldsymbol{\Psi}_{A}^{\kappa}, \\
\mathbf{K}^{\kappa}:=\frac{1}{2}\left(\gamma_{\mathbf{n}}^{-}+\gamma_{\mathbf{n}}^{+}\right) \operatorname{grad} \Psi_{V}^{\kappa}, & \mathbf{C}^{\kappa}:=\frac{1}{2}\left(\gamma_{D}^{-}+\gamma_{D}^{+}\right) \boldsymbol{\Psi}_{M}^{\kappa}, \\
\mathbf{A}^{\kappa}:=\frac{1}{2}\left(\gamma_{D}^{-}+\gamma_{D}^{+}\right) \Psi_{A}^{\kappa}, & \mathbf{N}^{\kappa}:=\frac{1}{2}\left(\gamma_{N}^{-}+\gamma_{N}^{+}\right) \boldsymbol{\Psi}_{M}^{\kappa},
\end{aligned}
$$

where $\gamma$ is the "standard trace" that amounts to pointwise restriction to $\Gamma$ of a scalar function. Note that the potentials are not necessarily continuous across $\Gamma$, which explains the need for averaging in the definitions of the boundary-integral operators. More precise information is provided by the jump relations, cf. [15, Section 5].

Theorem 3.4. The potentials satisfy the jump relations

$$
\begin{array}{ll}
{\left[\gamma \Psi_{V}^{\kappa}\right]_{\Gamma}=0,} & {\left[\left(\gamma_{\mathbf{n}} \circ \operatorname{grad}\right) \Psi_{V}^{\kappa}\right]_{\Gamma}=-I d,} \\
{\left[\gamma_{D} \Psi_{A}^{\kappa}\right]_{\Gamma}=0,} & {\left[\gamma_{N} \Psi_{A}^{\kappa}\right]_{\Gamma}=-I d,} \\
{\left[\gamma_{D} \boldsymbol{\Psi}_{M}^{\kappa}\right]_{\Gamma}=-I d,} & {\left[\gamma_{N} \Psi_{M}^{\kappa}\right]_{\Gamma}=0,} \\
{\left[\gamma_{\mathbf{n}} \boldsymbol{\Psi}_{A}^{\kappa}\right]_{\Gamma}=0,} & {\left[\gamma_{\mathbf{n}} \boldsymbol{\Psi}_{M}^{\kappa}\right]_{\Gamma}=0 .}
\end{array}
$$

Up to now, the meaning of the equalities in the jump relations is not entirely clear. Recall that even a smooth vector field will have discontinuous normal and tangential trace, if $\Gamma$ has edges and corners. This entails specifying appropriate function spaces (trace spaces) in which the equations are posed. 
The relevant function spaces are natural, because they are closely related to "energy norms". The most important norm is the electromagnetic energy norm of a vector field $\mathbf{U}$ on some domain $\Omega \subset \mathbb{R}^{3}$

$$
\|\mathbf{U}\|_{\boldsymbol{H}(\mathbf{c u r l} ; \Omega)}^{2}:=\int_{\Omega}|\mathbf{U}|^{2} \mathrm{~d} \mathbf{x}+\int_{\Omega}|\operatorname{curl} \mathbf{U}|^{2} \mathrm{~d} \mathbf{x} .
$$

By the usual completion procedure, it gives rise to the Hilbert space $\boldsymbol{H}(\mathbf{c u r l} ; \Omega)$ of vector fields with bounded electromagnetic energy. This induces a norm on the range of the electric trace operator $\gamma_{D}$

$$
\|\mathbf{u}\|_{\text {el }}:=\inf \left\{\|\mathbf{U}\|_{\boldsymbol{H}\left(\mathbf{c u r l} ; \Omega_{C}\right)}, \quad \mathbf{U} \in \boldsymbol{H}\left(\mathbf{c u r l} ; \Omega_{C}\right) \text { and } \gamma_{D}^{-} \mathbf{U}=\mathbf{u}\right\} .
$$

Let $\mathbf{X}_{\mathrm{el}}(\Gamma)$ stand for the Sobolev space (of distributions on $\Gamma$ ) induced by the norm $\|\cdot\|_{\mathrm{el}}$. This will be a trace space of "tangential vector fields", and $\gamma_{D}^{-}: \boldsymbol{H}\left(\mathbf{c u r l} ; \Omega_{C}\right) \mapsto \mathbf{X}_{\mathrm{el}}(\Gamma)$ will become a continuous and surjective operator. What is important about the norm from (18) is that it is intrinsic to $\Gamma$, that is, $\gamma_{D}^{+}: \boldsymbol{H}\left(\mathbf{c u r l} ; \Omega^{\prime}\right) \mapsto \mathbf{X}_{\mathrm{el}}(\Gamma)$ is continuous and surjective, too. More information about the underlying sophisticated mathematical theory can be found in [23,24] and the references cited therein. To deal with the magnetic trace, we introduce the inner product

$$
(\mathbf{u}, \mathbf{v})_{\tau}:=\int_{\Gamma} \mathbf{u} \cdot \overline{\mathbf{v}} \mathrm{d} S
$$

for complex valued tangential vector fields on $\Gamma$. A suitable norm for magnetic traces is the dual norm of $\|\cdot\|_{\mathrm{el}}$ :

$$
\|\varphi\|_{\text {mag }}:=\sup _{\mathbf{v} \in \mathbf{X}_{\mathrm{el}}(\Gamma)} \frac{(\boldsymbol{\varphi}, \mathbf{v})_{\boldsymbol{\tau}}}{\|\mathbf{v}\|_{\mathrm{el}}} .
$$

This norm will give rise to the Hilbert space $\mathbf{X}_{\operatorname{mag}}(\Gamma)$, which supplies the proper range space for $\gamma_{N}$ and also has an alternative intrinsic definition [23]. By definition, it is dual to $\mathbf{X}_{\mathrm{el}}(\Gamma)$ in the sense that

$$
\begin{array}{llll}
\boldsymbol{\varphi} \in \mathbf{X}_{\mathrm{mag}}(\Gamma): \quad(\boldsymbol{\varphi}, \mathbf{v})_{\boldsymbol{\tau}}=0 & \forall \mathbf{v} \in \mathbf{X}_{\mathrm{el}}(\Gamma) \quad \Rightarrow \quad \boldsymbol{\varphi}=0, \\
\mathbf{u} \in \mathbf{X}_{\mathrm{el}}(\Gamma): \quad(\boldsymbol{\mu}, \mathbf{v})_{\boldsymbol{\tau}}=0 \quad \forall \boldsymbol{\mu} \in \mathbf{X}_{\mathrm{mag}}(\Gamma) \quad \Rightarrow \quad \mathbf{u}=0 .
\end{array}
$$

In addition we mention that a similar duality holds for the trace operators $\gamma$ (on $H^{1}\left(\Omega_{C}\right)$ ) and $\gamma_{\mathbf{n}}\left(\right.$ on $\left.\boldsymbol{H}\left(\operatorname{div} ; \Omega_{C}\right)\right)$ and their associated trace spaces $H^{1 / 2}(\Gamma)$ and $H^{-1 / 2}(\Gamma)$ [20]. Here duality has to be understood with respect to the inner product

$$
(\varphi, \psi)_{0}:=\int_{\Gamma} \varphi \bar{\psi} \mathrm{d} S .
$$

Thus, we can give a rigorous meaning to the jump relations from Theorem 3.4. They are to be read in the sense of duality, i.e., with respect to integration over $\Gamma$ and testing with functions from the dual space.

Finally, for the sake of actual implementation, we need integral representations for the boundary-integral operators. It turns out that almost everywhere on $\Gamma$ these agree with the operators emerging from formally applying the traces to the potentials $\Psi_{A}^{\kappa}$ and $\Psi_{M}^{\kappa}$ as given in (13) and (14), respectively, e.g., for a tangential vector field $\lambda \in L^{\infty}(\Gamma)$

$$
\begin{aligned}
& \mathrm{A}^{\kappa}(\boldsymbol{\lambda})(\mathbf{x}):=\int_{\Gamma} G_{\kappa}(\mathbf{x}, \mathbf{y}) \lambda(\mathbf{y}) \mathrm{d} S(\mathbf{y}), \\
& \mathrm{B}^{\kappa}(\boldsymbol{\lambda})(\mathbf{x}):=\int_{\Gamma} \frac{\partial G_{\kappa}(\mathbf{x}, \mathbf{y})}{\partial \mathbf{n}(\mathbf{x})} \lambda(\mathbf{y})-\operatorname{grad}_{\mathbf{x}} G_{\kappa}(\mathbf{x}, \mathbf{y})(\boldsymbol{\lambda}(\mathbf{y}) \cdot \mathbf{n}(\mathbf{x})) \mathrm{d} S(\mathbf{y}),
\end{aligned}
$$

almost everywhere on $\Gamma$. 


\section{Coupled problem}

The jump relations from Theorem 3.4 tell us, how we can recover the value of interior/exterior traces of potentials. Thus, we get the Calderón identities: applying $\gamma_{D}^{-}$and $\gamma_{N}^{-}$to the representation formula of Theorem 3.2 we find for a solution $\mathbf{A}$ of (9),

$$
\begin{aligned}
& \gamma_{D}^{-} \mathbf{A}=\left(\mathbf{A}^{\kappa}-\frac{1}{\kappa^{2}} \operatorname{grad}_{\Gamma} \circ \mathbf{V}^{\kappa} \circ \operatorname{div}_{\Gamma}\right)\left(\gamma_{N}^{-} \mathbf{A}\right)+\left(\frac{1}{2} I d+\mathrm{C}^{\kappa}\right)\left(\gamma_{D}^{-} \mathbf{A}\right), \\
& \gamma_{N}^{-} \mathbf{A}=\left(\frac{1}{2} I d+\mathbf{B}^{\kappa}\right)\left(\gamma_{N}^{-} \mathbf{A}\right)+\mathrm{N}^{\kappa}\left(\gamma_{D}^{-} \mathbf{A}\right),
\end{aligned}
$$

where $\kappa=\frac{1}{2} \sqrt{2}(1+\mathrm{i}) \sqrt{\omega \sigma \mu_{c}}$. As the reaction field $\mathbf{U}$ satisfies the assumptions of Theorem 3.3, we conclude

$$
\begin{aligned}
& \gamma_{D}^{+} \mathbf{U}=-\mathbf{A}^{0}\left(\gamma_{N}^{+} \mathbf{U}\right)+\left(\frac{1}{2} I d-\mathbf{C}^{0}\right)\left(\gamma_{D}^{+} \mathbf{U}\right)-\operatorname{grad}_{\Gamma} \mathbf{V}^{0}\left(\gamma_{\mathbf{n}}^{+} \mathbf{U}\right), \\
& \gamma_{N}^{+} \mathbf{U}=\left(\frac{1}{2} I d-\mathbf{B}^{0}\right)\left(\gamma_{N}^{+} \mathbf{U}\right)-\mathbf{N}^{0}\left(\gamma_{D}^{+} \mathbf{U}\right), \\
& \gamma_{\mathbf{n}}^{+} \mathbf{U}=-\gamma_{\mathbf{n}}^{+} \Psi_{A}^{0}\left(\gamma_{N}^{+} \mathbf{U}\right)-\gamma_{\mathbf{n}}^{+} \Psi_{M}^{0}\left(\gamma_{D}^{+} \mathbf{U}\right)+\left(\frac{1}{2} I d-\mathbf{K}^{0}\right)\left(\gamma_{\mathbf{n}}^{+} \mathbf{U}\right) .
\end{aligned}
$$

This arises by applying $\gamma_{D}^{+}, \gamma_{N}^{+}$, and $\gamma_{\mathbf{n}}^{+}$to the exterior representation formula of Theorem 3.3. As we have stressed in the previous section, equalities in (21) and (22) have to be read in the respective trace spaces. To state results, it is useful to resort to the concept of Cauchy data:

Definition 4.1. Functions $\mathbf{v} \in \mathbf{X}_{\mathrm{el}}(\Gamma), \boldsymbol{\mu} \in \mathbf{X}_{\mathrm{mag}}(\Gamma)$ are called interior Cauchy data, if $\mathbf{v}=\gamma_{D}^{-} \mathbf{A}$, $\boldsymbol{\mu} \in \gamma_{N}^{-} \mathbf{A}$, where A solves (9). They represent exterior Cauchy data, if $\mathbf{v}=\gamma_{D}^{-} \mathbf{U}, \boldsymbol{\mu} \in \gamma_{N}^{-} \mathbf{U}$ with $\mathbf{U}$ satisfying the assumptions of Theorem 3.3.

Theorem 4.2. Traces $\gamma_{D}^{-} \mathbf{A}$ and $\gamma_{N}^{-} \mathbf{A}$ will be interior Cauchy data, if and only if they fulfill (21).

Traces $\gamma_{D}^{+} \mathbf{U}, \gamma_{N}^{+} \mathbf{U}$ will be exterior Cauchy data, if and only if they satisfy (22) and $\operatorname{div}_{\Gamma} \gamma_{N}^{+} \mathbf{U}=0$.

Proof. Firstly, it is clear from the derivation of (21) and (22) that traces of solutions A and $\mathbf{U}$ of (9) or (11), respectively, satisfy these equations. In the case of the second assertion, we point out that

$$
\text { curl curlU }=0, \quad \text { in } \Omega^{\prime} \Rightarrow \operatorname{div}_{\Gamma}\left(\gamma_{N}^{+} \mathbf{U}\right)=0
$$

which is an immediate consequence of the identity

$$
\operatorname{div}_{\Gamma}\left(\gamma_{D}^{+} \mathbf{V}\right)=\gamma_{\mathbf{n}}^{+}(\operatorname{curl} \mathbf{V})
$$

for the surface divergence $\operatorname{div}_{\Gamma}$.

On the other hand, if traces $\mathbf{v}:=\gamma_{D}^{-} \mathbf{A}, \boldsymbol{\mu}:=\gamma_{N}^{-} \mathbf{A}$ satisfy (21), Lemma 3.1 suggests that we simply use the representation formula of Theorem 3.2 to obtain a solution $\mathbf{A}$ of (9). By the definition of the boundary-integral operators and the jump relations from Theorem 3.4 we infer that its electric and magnetic traces will coincide with $\mathbf{v}$ and $\boldsymbol{\mu}$, respectively.

Further, if traces $(\mathbf{v}, \boldsymbol{\mu}, \varphi) \in \mathbf{X}_{\mathrm{el}}(\Gamma) \times \mathbf{X}_{\mathrm{mag}}(\Gamma) \times H^{-1 / 2}(\Gamma)$ with $\operatorname{div}_{\Gamma} \boldsymbol{\mu}=0$ solve (22), we resort to (16) and (17) for $\kappa=0$. This reveals that the representation formula of Theorem 3.3 gives the desired solution of (11). The decay conditions (4) are verified by straightforward inspection of the potentials. Then, appealing to the jump relations again completes the proof. 
The gist of the symmetric coupling approach according to Costabel [12] is to use all of the equations of the Calderon identities in conjunction with the transmission conditions (7). However, here we have to grapple with a mismatch of interior and exterior boundary data due to the presence of $\gamma_{\mathbf{n}}^{+} \mathbf{U}$ in (22). A remedy is motivated by the observation that the constraint $\operatorname{div}_{\Gamma}\left(\gamma_{N}^{+} \mathbf{U}\right)=0$ was essential in Theorem 4.2. In other words, $\gamma_{N}^{+} \mathbf{U}$ has to be sought in the space

$$
\mathbf{X}_{\mathrm{mag}}^{0}(\Gamma):=\left\{\boldsymbol{\mu} \in \mathbf{X}_{\mathrm{mag}}(\Gamma), \operatorname{div}_{\Gamma} \boldsymbol{\mu}=0\right\} .
$$

By the transmission condition for $\gamma_{N}$ and the fact that curlcurl| $\mathbf{A}_{s}=0$ in a neighborhood of $\Gamma, \gamma_{N}^{-} \mathbf{A}$ has to be $\operatorname{div}_{\Gamma}$-free, as well. Hence, we can restrict our attention to boundary data $\gamma_{N}^{-} \mathbf{A}, \gamma_{N}^{+} \mathbf{U} \in \mathbf{X}_{\text {mag }}^{0}(\Gamma)$ throughout.

This is a crucial hint on how to deal with the extra boundary data occurring in (22): recalling the dualities, we use $\mathbf{X}_{\text {mag }}^{0}(\Gamma)$ as test space for those equations of the Calderon identities that are set in $\mathbf{X}_{\mathrm{el}}(\Gamma)$. Since $\mathbf{X}_{\mathrm{mag}}^{0}(\Gamma)$ is a smaller space than $\mathbf{X}_{\mathrm{mag}}(\Gamma)$ this may involve a loss of information, but Cauchy data will definitely satisfy the resulting equations. The particular attraction of this option lies in the fact that

$$
\boldsymbol{\mu} \in \mathbf{X}_{\mathrm{mag}}^{0}(\Gamma) \Rightarrow\left(\boldsymbol{\mu}, \operatorname{grad}_{\Gamma} \varphi\right)_{\boldsymbol{\tau}}=0 \quad \forall \varphi \in H^{1 / 2}(\Gamma) .
$$

Conclusion (25) is straightforward, since $\operatorname{div}_{\Gamma}$ is the $(\cdot, \cdot)_{\tau}$-adjoint of $\operatorname{grad}_{\Gamma}$. This makes the undesirable terms disappear when switching to a weak form of the top equations in the Calderon identities (21) and (22)! For all $\boldsymbol{\mu} \in \mathbf{X}_{\mathrm{mag}}^{0}(\Gamma)$ we obtain

$$
\begin{aligned}
& (21) \Rightarrow\left(\boldsymbol{\mu}, \gamma_{D}^{-} \mathbf{A}\right)_{\boldsymbol{\tau}}=\left(\boldsymbol{\mu}, \mathrm{A}^{\kappa}\left(\gamma_{N}^{-} \mathbf{A}\right)\right)_{\boldsymbol{\tau}}+\left(\boldsymbol{\mu},\left(\frac{1}{2} I d+\mathrm{C}^{\kappa}\right) \gamma_{D}^{-} \mathbf{A}\right)_{\boldsymbol{\tau}}, \\
& (22) \Rightarrow\left(\boldsymbol{\mu}, \gamma_{D}^{+} \mathbf{U}\right)_{\boldsymbol{\tau}}=\left(\boldsymbol{\mu},-\mathrm{A}^{0}\left(\gamma_{N}^{+} \mathbf{U}\right)\right)_{\boldsymbol{\tau}}+\left(\boldsymbol{\mu},\left(\frac{1}{2} I d-\mathrm{C}^{0}\right) \gamma_{D}^{+} \mathbf{U}\right)_{\boldsymbol{\tau}} .
\end{aligned}
$$

Having obtained matching boundary data, we can combine the above equations and the second lines in (21) and (22) in a clever way. From the transmission conditions, we know $\gamma_{D}^{+} \mathbf{U}-$ $\gamma_{D}^{-} \mathbf{A}=-\gamma_{D}^{+} \mathbf{A}_{s}$. Thus, subtracting the above equations leads to

$$
\begin{aligned}
& -\left(\boldsymbol{\mu}, \mathrm{A}^{0}\left(\gamma_{N}^{+} \mathbf{U}\right)+\mathrm{A}^{\kappa}\left(\gamma_{N}^{-} \mathbf{A}\right)\right)_{\boldsymbol{\tau}}-\left(\boldsymbol{\mu}, \mathrm{C}^{0}\left(\gamma_{D}^{+} \mathbf{U}\right)+\mathrm{C}^{\kappa}\left(\gamma_{D}^{-} \mathbf{A}\right)\right)_{\boldsymbol{\tau}} \\
& \quad=-\frac{1}{2}\left(\boldsymbol{\mu}, \gamma_{D}^{+} \mathbf{A}_{s}\right)_{\boldsymbol{\tau}}
\end{aligned}
$$

for all $\boldsymbol{\mu} \in \mathbf{X}_{\mathrm{mag}}^{0}(\Gamma)$. From the transmission condition $1 / \mu_{0} \gamma_{N}^{+} \mathbf{U}-1 / \mu_{c} \gamma_{N}^{-} \mathbf{A}=-1 / \mu_{0} \gamma_{N}^{+} \mathbf{A}_{s}$ and the second equations of the Calderon identities, we directly conclude

$$
\begin{aligned}
& \frac{1}{\mu_{0}}\left(\frac{1}{2} I d-\mathrm{B}^{0}\right)\left(\gamma_{N}^{+} \mathbf{U}\right)-\frac{1}{\mu_{0}} \mathbf{N}^{0}\left(\gamma_{D}^{+} \mathbf{U}\right)-\frac{1}{\mu_{c}}\left(\frac{1}{2} I d+\mathrm{B}^{\kappa}\right)\left(\gamma_{N}^{-} \mathbf{A}\right)-\frac{1}{\mu_{c}} \mathbf{N}^{\kappa}\left(\gamma_{D}^{-} \mathbf{A}\right) \\
& \quad=-\frac{1}{\mu_{0}} \gamma_{N}^{+} \mathbf{A}_{s} .
\end{aligned}
$$

As final unknown quantities we introduce the tangential trace of the vector potential $\mathbf{u}:=$ $\gamma_{D}^{-} \mathbf{A} \in \mathbf{X}_{\mathrm{el}}(\Gamma)$ and the tangential trace of the magnetic field $\lambda:=\frac{1}{\mu_{c}} \gamma_{N}^{-} \mathbf{A} \in \mathbf{X}_{\mathrm{mag}}^{0}(\Gamma)$. The latter is also known as the equivalent surface current. The transmission conditions enable us to express the exterior traces in these unknowns. We end up with the coupled variational problem: Seek $\mathbf{u} \in \mathbf{X}_{\mathrm{el}}(\Gamma), \lambda \in \mathbf{X}_{\mathrm{mag}}^{0}(\Gamma)$ such that

$$
\begin{aligned}
& -\left(\left(\frac{1}{\mu_{0}} \mathrm{~N}^{0}+\frac{1}{\mu_{c}} \mathrm{~N}^{\kappa}\right) \mathbf{u}, \mathbf{v}\right)_{\tau}-\left(\left(\mathrm{B}^{0}+\mathrm{B}^{\kappa}\right) \lambda, \mathbf{v}\right)_{\tau}=f(\mathbf{v}), \\
& -\left(\boldsymbol{\mu},\left(\mathrm{C}^{0}+\mathrm{C}^{\kappa}\right) \mathbf{u}\right)_{\tau}+\left(\boldsymbol{\mu},\left(\mu_{0} \mathrm{~A}^{0}+\mu_{c} \mathrm{~A}^{\kappa}\right) \lambda\right)_{\tau}=g(\boldsymbol{\mu})
\end{aligned}
$$


for all $\mathbf{v} \in \mathbf{X}_{\mathrm{el}}(\Gamma), \boldsymbol{\mu} \in \mathbf{X}_{\mathrm{mag}}^{0}(\Gamma)$. The right-hand side is given by

$$
\begin{aligned}
& f(\mathbf{v}):=-\frac{1}{\mu_{0}}\left(\left(\frac{1}{2} I d+\mathrm{B}^{0}\right) \gamma_{N}^{+} \mathbf{A}_{s}, \mathbf{v}\right)_{\boldsymbol{\tau}}-\frac{1}{\mu_{0}}\left(\mathrm{~N}^{0}\left(\gamma_{D}^{+} \mathbf{A}_{s}\right), \mathbf{v}\right)_{\boldsymbol{\tau}}, \\
& g(\boldsymbol{\mu}):=\left(\boldsymbol{\mu},\left(\frac{1}{2} I d+\mathrm{C}^{0}\right) \gamma_{D}^{+} \mathbf{A}_{s}\right)_{\boldsymbol{\tau}}+\left(\boldsymbol{\mu}, \mathrm{A}^{0}\left(\gamma_{N}^{+} \mathbf{A}_{s}\right)\right)_{\boldsymbol{\tau}} .
\end{aligned}
$$

The analysis for the variational problem (28) relies on two profound results. The first concerns the symmetry of boundary-integral operators [25, Theorem 3.9], [15, Section 6].

Theorem 4.3. If $\mathfrak{R e} \kappa^{2} \geq 0$, the boundary integral operators $\mathrm{B}^{\kappa}$ and $\mathrm{C}^{\kappa}$ satisfy

$$
\left(\mathrm{B}^{\kappa} \boldsymbol{\mu}, \mathbf{v}\right)_{\boldsymbol{\tau}}=-\left(\boldsymbol{\mu}, \mathrm{C}^{\kappa} \mathbf{v}\right)_{\boldsymbol{\tau}} \quad \forall \boldsymbol{\mu} \in \mathbf{X}_{\mathrm{mag}}(\Gamma), \quad \mathbf{v} \in \mathbf{X}_{\mathrm{el}}(\Gamma) .
$$

The second result is a statement about the ellipticity of some boundary-integral operators with respect to the trace norms, see [26, Proposition.4.1] and [20, Corollary 8.13]

Theorem 4.4. If $\mathfrak{R e} \kappa^{2} \geq 0$, the following estimates hold true

$$
\begin{array}{lll}
\mathfrak{R e}\left\{\left(\boldsymbol{\mu}, \mathrm{A}^{\kappa} \boldsymbol{\mu}\right)_{\boldsymbol{\tau}}\right\} \geq c\|\boldsymbol{\mu}\|_{\mathrm{mag}}^{2}, & \mathfrak{I m}\left\{\left(\boldsymbol{\mu}, \mathrm{A}^{\kappa} \boldsymbol{\mu}\right)_{\boldsymbol{\tau}}\right\} \geq 0 & \forall \boldsymbol{\mu} \in \mathbf{X}_{\mathrm{mag}}^{0}(\Gamma), \\
\mathfrak{R e}\left\{\left(\varphi, \mathrm{V}^{\kappa} \varphi\right)_{0}\right\} \geq c\|\varphi\|_{H^{-1 / 2}(\Gamma)}^{2}, & \mathfrak{I m}\left\{\left(\varphi, \mathrm{V}^{\kappa} \varphi\right)_{0}\right\} \geq 0 & \forall \varphi \in H^{-1 / 2} \Gamma,
\end{array}
$$

with (possibly different) positive constants $c>0$ depending on $\kappa$ and $\Gamma$.

The proof of these inequalities follows the same lines as the proof of the next theorem.

Theorem 4.5. If $\kappa^{2}=i \rho$ with $\rho>0$, then

$$
\mathfrak{R e}\left\{-\left(\mathrm{N}^{\kappa} \mathbf{v}, \mathbf{v}\right)_{\boldsymbol{\tau}}\right\} \geq 0, \quad \mathfrak{I m}\left\{-\left(\mathrm{N}^{\kappa} \mathbf{v}, \mathbf{v}\right)_{\boldsymbol{\tau}}\right\} \geq 0 \quad \forall \mathbf{v} \in \mathbf{X}_{\mathrm{el}}(\Gamma),
$$

and there is $c=c(\kappa, \Gamma)>0$ such that

$$
\left|-\left(\mathrm{N}^{\kappa} \mathbf{v}, \mathbf{v}\right)_{\boldsymbol{\tau}}\right| \geq c\|\mathbf{v}\|_{\mathrm{el}}^{2} \quad \forall \mathbf{v} \in \mathbf{X}_{\mathrm{el}}(\Gamma) .
$$

Proof. Pick $\mathbf{v} \in \mathbf{X}_{\mathrm{el}}(\Gamma)$. Then, by the jump relation from Theorem. 3.4, the fact that $\mathbf{V}:=$ $\boldsymbol{\Psi}_{M}^{\kappa}$ (v) fulfills curl curl $\mathbf{V}+\kappa^{2} \mathbf{V}=0$ in $\Omega_{C} \cup \Omega^{\prime}$, see Lemma 3.1, and the integration by parts formula for the curl-operator

$$
\begin{aligned}
-\left(\mathrm{N}^{\kappa} \mathbf{v}, \mathbf{v}\right)_{\boldsymbol{\tau}} & =\left(\gamma_{N} \Psi_{M}^{\kappa}(\mathbf{v}),\left[\gamma_{D} \Psi_{M}^{\kappa}(\mathbf{v})\right]_{\Gamma}\right)_{\boldsymbol{\tau}} \\
& =\int_{\mathbb{R}^{3} \backslash \Gamma} \mid \text { curl }\left.\mathbf{V}\right|^{2}-\operatorname{curl} \operatorname{curl} \mathbf{V} \cdot \overline{\mathbf{V}} \mathrm{d} \mathbf{x} \\
& =\int_{\mathbb{R}^{3} \backslash \Gamma}|\operatorname{curl} \mathbf{V}|^{2}+\kappa^{2}|\mathbf{V}|^{2} \mathrm{~d} \mathbf{x}
\end{aligned}
$$

This identity yields the first assertion of the theorem. The second follows from the continuity of the trace $\gamma_{D}$ : for $c>0$ independent of $\mathbf{v}$,

$$
\|\mathbf{v}\|_{\mathrm{el}}=\left\|[\mathbf{V}]_{\Gamma}\right\|_{\mathrm{el}} \leq c\|\mathbf{v}\|_{\boldsymbol{H}\left(\mathbf{c u r l} ; \Omega_{C} \cup \Omega^{\prime}\right)} .
$$

Moreover, [2, Formula (2.86)] provides us with the identity

$$
\left(\mathrm{N}^{\kappa} \mathbf{u}, \mathbf{v}\right)_{\boldsymbol{\tau}}=-\kappa^{2}\left(\mathrm{~A}^{\kappa}(\mathbf{u} \times \mathbf{n}), \mathbf{v} \times \mathbf{n}\right)_{\boldsymbol{\tau}}-\left(\mathrm{V}^{\kappa}\left(\operatorname{curl}_{\Gamma} \mathbf{u}\right), \operatorname{curl}_{\Gamma} \mathbf{v}\right)_{0} .
$$


Now we are in a position to prove the main result about the bilinear form

$$
\begin{aligned}
\mathbf{d}\left(\left(\begin{array}{c}
\mathbf{u} \\
\boldsymbol{\lambda}
\end{array}\right),\left(\begin{array}{c}
\mathbf{v} \\
\boldsymbol{\mu}
\end{array}\right)\right)= & -\left(\left(\frac{1}{\mu_{0}} \mathrm{~N}^{0}+\frac{1}{\mu_{c}} \mathrm{~N}^{\kappa}\right) \mathbf{u}, \mathbf{v}\right)_{\boldsymbol{\tau}}-\left(\left(\mathrm{B}^{0}+\mathrm{B}^{\kappa}\right) \boldsymbol{\lambda}, \mathbf{v}\right)_{\boldsymbol{\tau}} \\
& -\left(\boldsymbol{\mu},\left(\mathrm{C}^{0}+\mathrm{C}^{\kappa}\right) \mathbf{u}\right)_{\boldsymbol{\tau}}+\left(\boldsymbol{\mu},\left(\mu_{0} \mathrm{~A}^{0}+\mu_{c} \mathrm{~A}^{\kappa}\right) \boldsymbol{\lambda}\right)_{\boldsymbol{\tau}},
\end{aligned}
$$

underlying the coupled variational formulation (28).

Theorem 4.6. The bilinear form $\mathbf{d}$ associated with the variational problem (28) is $\mathbf{X}_{\mathrm{el}}(\Gamma) \times$ $\mathbf{X}_{\mathrm{mag}}^{0}(\Gamma)$-elliptic in the sense that there is $c>0$ such that

$$
\left|\mathbf{d}\left(\left(\begin{array}{c}
\mathbf{v} \\
\boldsymbol{\mu}
\end{array}\right),\left(\begin{array}{c}
\mathbf{v} \\
\boldsymbol{\mu}
\end{array}\right)\right)\right| \geq c\left\{\|\mathbf{v}\|_{\mathrm{el}}^{2}+\|\boldsymbol{\mu}\|_{\mathrm{mag}}^{2}\right\}
$$

for all $\mathbf{v} \in \mathbf{X}_{\mathrm{el}}(\Gamma)$ and $\boldsymbol{\mu} \in \mathbf{X}_{\mathrm{mag}}(\Gamma)$.

Proof. As a simple consequence of the block skew-symmetric structure of the variational problem (see Theorem 4.3) we find for $\mathbf{v} \in \mathbf{X}_{\mathrm{el}}(\Gamma), \boldsymbol{\mu} \in \mathbf{X}_{\mathrm{mag}}^{0}(\Gamma)$ that

$$
\mathbf{d}\left(\left(\begin{array}{c}
\mathbf{v} \\
\boldsymbol{\mu}
\end{array}\right),\left(\begin{array}{c}
\mathbf{v} \\
\boldsymbol{\mu}
\end{array}\right)\right)=-\left(\left(\frac{1}{\mu_{0}} \mathrm{~N}^{0}+\frac{1}{\mu_{c}} \mathrm{~N}^{\kappa}\right) \mathbf{v}, \mathbf{v}\right)_{\boldsymbol{\tau}}+\left(\boldsymbol{\mu},\left(\mu_{0} \mathrm{~A}^{0}+\mu_{c} \mathrm{~A}^{\kappa}\right) \boldsymbol{\mu}\right)_{\boldsymbol{\tau}} .
$$

By Theorems 4.4 and 4.5, the real and imaginary parts of all summands are positive. Hence,

$$
\begin{aligned}
\left|\mathbf{d}\left(\left(\begin{array}{c}
\mathbf{v} \\
\boldsymbol{\mu}
\end{array}\right),\left(\begin{array}{c}
\mathbf{v} \\
\boldsymbol{\mu}
\end{array}\right)\right)\right|^{2} \geq & \left|-\frac{1}{\mu_{0}}\left(\mathrm{~N}^{0} \mathbf{v}, \mathbf{v}\right)_{\boldsymbol{\tau}}\right|^{2}+\left|-\frac{1}{\mu_{c}}\left(\mathrm{~N}^{\kappa} \mathbf{v}, \mathbf{v}\right)_{\boldsymbol{\tau}}\right|^{2} \\
& +\left|\mu_{0}\left(\boldsymbol{\mu}, \mathbf{A}^{0} \boldsymbol{\mu}\right)_{\boldsymbol{\tau}}\right|^{2}+\left|\mu_{c}\left(\boldsymbol{\mu}, \mathbf{A}^{\kappa} \boldsymbol{\mu}\right)_{\boldsymbol{\tau}}\right|^{2} .
\end{aligned}
$$

For the first term, we can rely on (30). The others are taken care of by the ellipticity estimates of Theorems 4.4 and 4.5 .

Theorem 4.7. The variational problem (28-29) has a unique solution $(\mathbf{u}, \lambda) \in \mathbf{X}_{\mathrm{el}}(\Gamma) \times \mathbf{X}_{\mathrm{mag}}^{0}(\Gamma)$, which coincides with $\left(\gamma_{D}^{-} \mathbf{A}, 1 / \mu_{c} \gamma_{N}^{-} \mathbf{A}\right)$ for the solution $\mathbf{A}$ of (9-10).

Proof. Existence and uniqueness of solutions is immediate from Theorem 4.6 and the LaxMilgram Lemma [20, Lemma 2.32].

By the derivation of the boundary-integral equations it is clear that, given a solution $(\mathbf{A}, \mathbf{U})$ of (9-10), the traces $\mathbf{u}:=\gamma_{D}^{-} \mathbf{A}, \lambda:=1 / \mu_{c} \gamma_{N}^{-} \mathbf{A}$ will satisfy (28-29). Existence and uniqueness of solutions of both (9-10) and (28-29) complete the proof.

In the case of impedance boundary conditions (12) the coupling strategy is slightly different. We start from the exterior Calderón projector in weak form

$$
\begin{aligned}
& \left(\gamma_{N}^{+} \mathbf{U}, \mathbf{v}\right)_{\boldsymbol{\tau}}=\left(\left(\frac{1}{2} I d-\mathrm{B}^{0}\right)\left(\gamma_{N}^{+} \mathbf{U}\right), \mathbf{v}\right)_{\boldsymbol{\tau}}-\left(\mathrm{N}^{0}\left(\gamma_{D}^{+} \mathbf{U}\right), \mathbf{v}\right)_{\boldsymbol{\tau}}, \\
& \left(\boldsymbol{\mu}, \gamma_{D}^{+} \mathbf{U}\right)_{\boldsymbol{\tau}}=\left(\boldsymbol{\mu},-\mathrm{A}^{0}\left(\gamma_{N}^{+} \mathbf{U}\right)\right)_{\boldsymbol{\tau}}+\left(\boldsymbol{\mu},\left(\frac{1}{2} I d-\mathrm{C}^{0}\right) \gamma_{D}^{+} \mathbf{U}\right)_{\boldsymbol{\tau}},
\end{aligned}
$$

for all $\mathbf{v} \in \mathbf{X}_{\mathrm{el}}(\Gamma), \boldsymbol{\mu} \in \mathbf{X}_{\mathrm{mag}}^{0}(\Gamma)$, and use the transmission conditions (10). As before, $\mathbf{u}:=\gamma_{D}^{-} \mathbf{A} \in$ $\mathbf{X}_{\mathrm{el}}(\Gamma)$ and $\lambda:=1 / \mu_{c} \gamma_{N}^{-} \mathbf{A} \in \mathbf{X}_{\text {mag }}^{0}(\Gamma)$ will be retained as unknowns. In addition, the impedance boundary conditions will be used to express $\gamma_{N}^{+} \mathbf{U}=-\gamma_{N}^{+} \mathbf{A}_{s}+\mu_{0} \mu_{c}^{-1} \eta^{-1} \mathbf{u}$ on the lefthand side of the upper equation. This yields the variational problem: seek $\mathbf{u} \in \mathbf{X}_{\mathrm{el}}(\Gamma) \cap \boldsymbol{L}^{2}(\Gamma)$, $\lambda \in \mathbf{X}_{\mathrm{mag}}(\Gamma)$ such that 


$$
\begin{aligned}
& \left(\frac{1}{\mu_{c} \eta} \mathbf{u}+\frac{1}{\mu_{0}} \mathrm{~N}^{0} \mathbf{u}, \mathbf{v}\right)_{\boldsymbol{\tau}}-\left(\left(\frac{1}{2} I d-\mathrm{B}^{0}\right) \lambda, \mathbf{v}\right)_{\boldsymbol{\tau}}=\ldots, \\
& \left(\boldsymbol{\mu},\left(\frac{1}{2} I d+\mathrm{C}^{0}\right) \mathbf{u}\right)_{\boldsymbol{\tau}}+\mu_{0}\left(\boldsymbol{\mu}, \mathrm{A}^{0} \lambda\right)_{\tau}=\ldots
\end{aligned}
$$

for all $\mathbf{v} \in \mathbf{X}_{\mathrm{el}}(\Gamma) \cap \boldsymbol{L}^{2}(\Gamma), \boldsymbol{\mu} \in \mathbf{X}_{\mathrm{mag}}(\Gamma)$. The functionals on the right-hand side have been omitted, since they are straightforward.

Note the enhanced regularity required from $\mathbf{u}, \mathbf{v}$. Thanks to a result from [27] that asserts the continuity of $\mathbf{N}^{0}: \mathbf{X}_{\mathrm{el}}(\Gamma) \cap \boldsymbol{L}^{2}(\Gamma) \mapsto \mathbf{X}_{\mathrm{mag}}(\Gamma)$ and $\mathrm{C}^{0}: \mathbf{X}_{\mathrm{el}}(\Gamma) \cap \boldsymbol{L}^{2}(\Gamma) \mapsto \mathbf{X}_{\mathrm{el}}(\Gamma) \cap \boldsymbol{L}^{2}(\Gamma)$, the variational problem (31) can be lifted to the function space $\mathbf{X}_{\mathrm{el}}(\Gamma) \cap \boldsymbol{L}^{2}(\Gamma)$. Moreover, the apparent block skew-symmetric structure implies ellipticity on $\left(\mathbf{X}_{\mathrm{el}}(\Gamma) \cap \boldsymbol{L}^{2}(\Gamma)\right) \times \mathbf{X}_{\mathrm{mag}}(\Gamma)$ by analogy with Theorem 4.6.

\section{Galerkin discretization}

We select a conforming Galerkin boundary-element discretization of (28) and (29) that relies on finite dimensional subspaces $\mathcal{W}_{h} \subset \mathbf{X}_{\mathrm{el}}(\Gamma)$ and $\mathcal{V}_{h} \subset \mathbf{X}_{\text {mag }}^{0}(\Gamma)$. These should be boundaryelement spaces in the sense that

1. the functions in both $\mathcal{W}_{h}$ and $\mathcal{V}_{h}$ are piecewise polynomial tangential vector fields with respect to a mesh $\Gamma_{h}$ of $\Gamma$ consisting of flat triangles;

2. there are bases of $\mathcal{W}_{h}$ and $\mathcal{V}_{h}$ that only comprise locally supported functions.

For the construction of $\mathcal{W}_{h}$ we start from $\boldsymbol{H}\left(\mathbf{c u r l} ; \Omega_{C}\right)$-conforming finite-element schemes for the approximation of vector potentials. The simplest is provided by the so-called edge elements [28]. Keeping in mind that $\mathbf{X}_{\mathrm{el}}(\Gamma):=\gamma_{D}\left(\boldsymbol{H}\left(\mathbf{c u r l} ; \Omega_{C}\right)\right)$, we simply take the tangential projections of edge-element functions on a mesh $\Omega_{h}$ with $\Omega_{h \mid \Gamma}=\Gamma_{h}$ as space $\mathcal{W}_{h}$. This will give a space of piecewise linear vector fields on $\Gamma$, whose tangential components are continuous across edges of triangles. This is a well-known sufficient condition for $\mathcal{W}_{h} \subset \mathbf{X}_{\mathrm{el}}(\Gamma)$. The local shape functions on a triangle $T$ are given by the formula

$$
\mathbf{b}_{i, j}^{T}:=\lambda_{i} \operatorname{grad}_{\Gamma} \lambda_{j}-\lambda_{j} \operatorname{grad}_{\Gamma} \lambda_{i} \quad 1 \leq i<j \leq 3,
$$

where $\lambda_{i}, i=1,2,3$, are the local linear barycentric coordinate functions in $T$. These basis functions are sketched in Figure 1. They are associated with the edges of $\Gamma_{h}$ so that $\operatorname{dim} \mathcal{W}_{h}$ will agree with the total number of edges of $\Gamma_{h}$. Note that $\mathcal{W}_{h}$ can also be obtained by $90^{\circ}$ rotation of the lowest order div-conforming Raviart-Thomas elements in 2D, cf. [29, Chapter 3]. More details can be found in [30, Section 2.2].

In order to find $\mathcal{V}_{h}$ we recall that $\lambda$ is the rotated tangential trace of the magnetic field $\mathbf{H}$. As $\boldsymbol{H}(\mathbf{c u r l} ; \Omega)$ is the right function space for $\mathbf{H}$, too, we get the right boundary-element space for magnetic traces by rotating functions in $\mathcal{W}_{h}$ by $90^{\circ}$. This will give surface vector fields

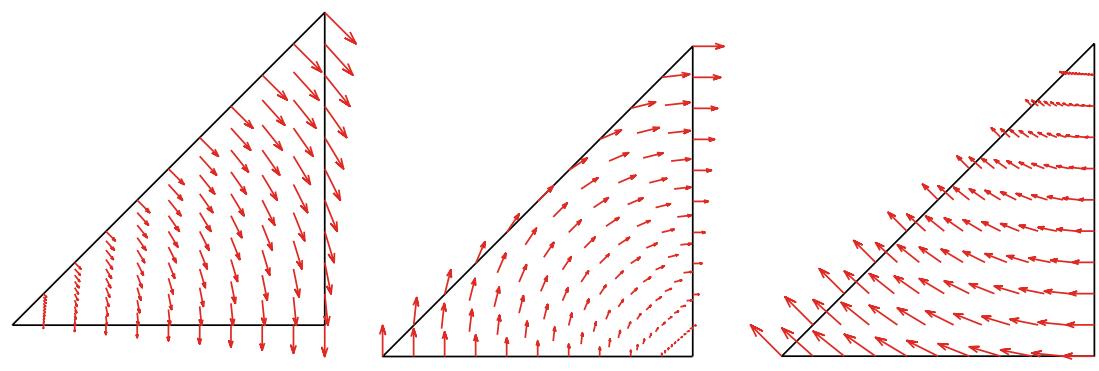

Figure 1. Local shape functions of $\mathcal{W}_{h}$. 
with continuous fluxes across edges of triangles, which is a very desirable property for discrete equivalent surface currents. However, ellipticity of (28) only holds provided that $\operatorname{div}_{\Gamma} \lambda=0$. Therefore, this property has to be enforced on $\mathcal{V}_{h}$. Formally, we may choose

$$
\mathcal{V}_{h}:=\left\{\boldsymbol{\mu}_{h} \in \mathcal{W}_{h} \times \mathbf{n}, \operatorname{div}_{\Gamma} \boldsymbol{\mu}=0\right\} .
$$

Using the formula (32), we readily see that $\mathcal{V}_{h}$ only contains piecewise constant vector fields.

By Theorem 4.6 and Cea's lemma [31, Theorem 2.4.1] conformity of the Galerkin method directly translates into the quasi-optimal error estimate in energy norm

$$
\left\|\mathbf{u}-\mathbf{u}_{h}\right\|_{\mathrm{el}}+\left\|\lambda-\lambda_{h}\right\|_{\mathrm{mag}} \leq C\left(\inf _{\mathbf{v}_{h} \in \mathcal{W}}\left\|\mathbf{u}-\mathbf{v}_{h}\right\|_{\mathrm{el}}+\inf _{\zeta_{h} \in \mathcal{V}_{h}}\left\|\lambda-\zeta_{h}\right\|_{\mathrm{mag}}\right),
$$

where $\mathbf{u}_{h}$ and $\lambda_{h}$ stand for the boundary-element solutions, and $C>0$ depends on the ellipticity and continuity constants of the continuous variational problem (28). Hence, approximate error estimates for the finite-element spaces will directly provide us with rates of convergence. Let us assume quasi-uniform and shape regular families of surface meshes $\Gamma_{h}$, where $h$ denotes the meshwidth. Provided that the continuous solutions $\mathbf{u}$ and $\lambda$ are sufficiently smooth, we arrive at

$$
\left\|\mathbf{u}-\mathbf{u}_{h}\right\|_{\mathrm{el}}+\left\|\boldsymbol{\lambda}-\boldsymbol{\lambda}_{h}\right\|_{\mathrm{mag}} \leq C\left(h^{\min \left\{\frac{3}{2}, \eta+\frac{1}{2}\right\}}\|\mathbf{u}\|_{\boldsymbol{H}^{\eta}\left(\operatorname{curl}_{\Gamma}, \Gamma\right)}+h^{\min \left\{\frac{3}{2}, \rho+\frac{1}{2}\right\}}\|\boldsymbol{\lambda}\|_{\boldsymbol{H}^{\rho}(\Gamma)}\right),
$$

for some $\eta, \rho>0$. The constant $C>0$ now depends on the shape-regularity of the meshes, too. Details about approximation by functions in $\mathcal{W}_{h}$ can be found in [32, Section 4.2.2]. The possible ranges of $\eta$ and $\rho$ depend on the geometry of $\Gamma$ : the presence of edges and corners will impose limits on $\eta, \rho$. At worst, these may only be slightly larger than zero.

Due to the divergence constraint in $\mathcal{V}_{h}$ we cannot use rotated shape functions from Figure 1 to get a locally supported basis. A remedy is offered by using scalar surface stream functions. Let $\mathcal{S}_{h}$ stand for the space of $\Gamma_{h}$-piecewise linear and continuous functions on $\Gamma$. Then, if $\Gamma$ is simply connected, we know from deRham's theorem [28, Corollary 3.3] that $\mathcal{V}_{h}=\operatorname{curl}_{\Gamma} \mathcal{S}_{h}$. Hence, we may simply use the surface rotation of the "hat basis functions" of $\mathcal{S}_{h}$ as a basis for $\mathcal{V}_{h}$, see Figure 2 (left).

Because we have not ruled out more general topologies of $\Gamma$, surface co-homology vector fields can also contribute to the kernel of $\operatorname{div}_{\Gamma}$ :

$$
\mathcal{V}_{h}=\operatorname{curl}_{\Gamma} \mathcal{S}_{h} \oplus \mathcal{H}_{h}, \quad \operatorname{dim} \mathcal{H}_{h}=\beta_{1}(\Gamma),
$$

where $\beta_{1}(\Gamma)$ is the first Betti number of $\Gamma$, which is twice the number of holes drilled through $\Omega_{C}$. This means that $\operatorname{dim} \mathcal{V}_{h}$ will be equal to the number of vertices of $\Gamma_{h}$ plus $\beta_{1}(\Gamma)$.
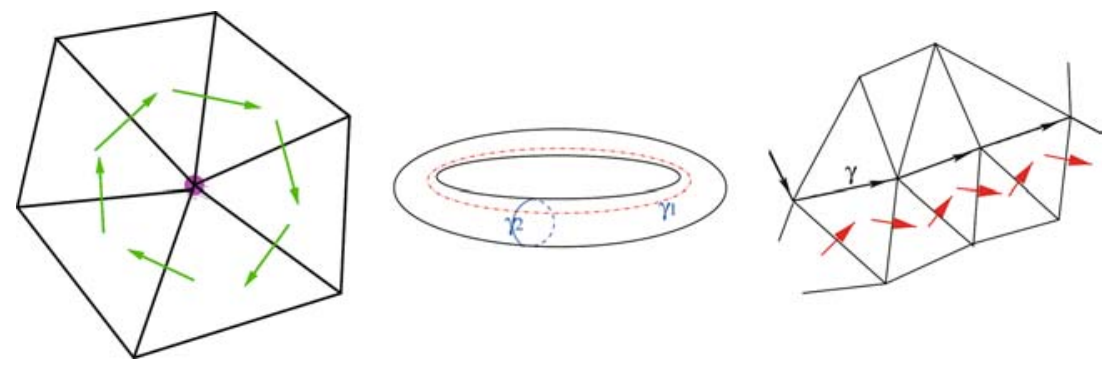

Figure 2. Basis function of $\mathcal{V}_{h}$ associated with a vertex (left). Fundamental cycles for the torus (middle). Current street along a section of a path $\gamma$ (right). 


\section{R. Hiptmair and J. Ostrowski}

To find a basis of $\mathcal{H}_{h}$ we need representatives $\gamma_{k}, k=1 \ldots, \beta_{1}(\Gamma)$, of a basis of the cohomology group $H_{1}\left(\Gamma_{h}, \mathbb{Z}\right)$ in the form of oriented closed edge paths (cycles). In other words, we need a maximal set of closed curves on the surface that do not cut the surface into two separate parts, and cannot be deformed into each other by sweeping them over parts of $\Gamma$. Typical choices for the torus are depicted in Figure 2 (middle). We can always find such curves that run along edges of $\Gamma_{h}$. To each such path $\gamma$ a "current sheet" $\eta_{\gamma}$ can be associated, a circular current traveling along the path, see Figure 2 (right).

Consider a non-bounding surface edge cycle $\gamma$ that is bounding with respect to $\Omega_{e}$, that is, there is an oriented surface $\Sigma \subset \Omega_{e}$ such that $\gamma=\partial \Sigma$. Then we get from Stokes theorem

$$
\int_{\gamma}\left(\gamma_{N} \mathbf{A} \times \mathbf{n}\right) \cdot \mathrm{d} \vec{s}=\int_{\Sigma} \operatorname{curl} \operatorname{curl} \mathbf{A} \cdot \mathbf{n} \mathrm{d} S=0 .
$$

As curl curl $\mathbf{A}=0$ in $\Omega_{e}$, this means that, in the discrete variational problem (36), we can confine ourselves to those $\lambda_{h} \in \mathcal{V}_{h}$ that satisfy $\int_{\gamma}\left(\lambda_{h} \times \mathbf{n}\right) \cdot \mathrm{d} \vec{s}=0$ for all cycles $\gamma$ bounding relative to $\Omega_{e}$. This means that we only have to take into account current sheets along cycles bounding relative to the exterior. An algorithm for the construction of these cycles has been developed in [33]. The resulting basis of the relevant subspace of $\mathcal{H}_{h}$ will be denoted by $\iota_{1}, \ldots, \iota_{L}$, $L=: \frac{1}{2} \beta_{1}(\Gamma)$. Then the discrete linear variational problem arising from (28) reads: search for $\mathbf{u}_{h} \in \mathcal{W}_{h}, \varphi_{h} \in \mathcal{S}_{h} / \mathbb{R},\left(\alpha_{1}, \ldots, \alpha_{L}\right)^{T} \in \mathbb{C}^{L}$ such that

$$
\begin{aligned}
& -\left(\tilde{\mathrm{N}} \mathbf{u}_{h}, \mathbf{v}_{h}\right)_{\boldsymbol{\tau}}-\left(\tilde{\mathrm{B}} \operatorname{curl}_{\Gamma} \varphi_{h}, \mathbf{v}_{h}\right)_{\tau}-\sum_{k=1}^{L} \alpha_{k}\left(\tilde{\mathrm{B}} \iota^{k}, \mathbf{v}_{h}\right)_{\boldsymbol{\tau}}=f\left(\mathbf{v}_{h}\right), \\
& \left(\tilde{\mathrm{B}} \operatorname{curl}{ }_{\Gamma} \psi_{h}, \mathbf{u}_{h}\right)_{\boldsymbol{\tau}}+\left(\operatorname{curl}_{\Gamma} \psi_{h}, \tilde{\mathrm{A}} \operatorname{curl} \varphi_{\Gamma}\right)_{\tau}+\sum_{k=1}^{L} \alpha_{k}\left(\operatorname{curl}_{\Gamma} \psi_{h}, \tilde{\mathrm{A}} \iota_{k}\right)_{\boldsymbol{\tau}}=g\left(\operatorname{curl}_{\Gamma} \psi_{h}\right), \\
& \left(\tilde{\mathrm{B}} \boldsymbol{\iota}^{j}, \mathbf{u}_{h}\right)_{\boldsymbol{\tau}}+\left(\boldsymbol{\iota}^{j}, \tilde{\mathrm{A}} \operatorname{curl}_{\Gamma} \varphi_{h}\right)_{\boldsymbol{\tau}}+\sum_{k=1}^{L} \alpha_{k}\left(\boldsymbol{\iota}^{j}, \tilde{\mathrm{A}} \boldsymbol{\iota}_{k}\right)_{\boldsymbol{\tau}}=g\left(\boldsymbol{\iota}^{j}\right),
\end{aligned}
$$

for all $\mathbf{v}_{h} \in \mathcal{W}_{h}, \psi_{h} \in \mathcal{S}_{h} / \mathbb{R}, j=1, \ldots, L$. We abbreviated $\tilde{\mathrm{A}}:=\mu_{0} \mathrm{~A}^{0}+\mu_{c} \mathrm{~A}^{\kappa}, \tilde{\mathrm{B}}=\mathrm{B}^{0}+\mathrm{B}^{\kappa}, \tilde{\mathrm{N}}:=$ $1 / \mu_{0} \mathrm{~N}^{0}+1 / \mu_{c} \mathrm{~N}^{\kappa}$. From (36) we can retrieve $\lambda_{h}=\operatorname{curl}_{\Gamma} \varphi_{h}+\sum_{k=1}^{L} \alpha_{k} l^{k}$. We point out that in the case of impedance boundary conditions, that is, for the variational problem (31), Galerkin discretization can be accomplished in exactly the same fashion.

\section{Implementation}

The first algorithmic issue concerns the evaluation of expressions of the form $(\mathbf{X} \cdot, \cdot)_{\tau}$, where $\mathrm{X}$ is a boundary-integral operator and the arguments will be basis functions from either $\mathcal{W}_{h}$ or $\mathcal{V}_{h}$. In detail, as a consequence of (30), we have to compute

$$
\left(\mathrm{V}^{\kappa} \varphi_{h}, \psi_{h}\right)_{0}=\int_{\Gamma} \int_{\Gamma} G_{\kappa}(\mathbf{x}, \mathbf{y}) \varphi_{h}(\mathbf{x}) \psi_{h}(\mathbf{y}) \mathrm{d} S(\mathbf{x}) \mathrm{d} S(\mathbf{y})
$$

for piecewise constant functions $\varphi_{h}$ and $\psi_{h}$, the similar expressions

$$
\left(\mathrm{A}^{\kappa} \varphi_{h}, \psi_{h}\right)_{\tau}=\int_{\Gamma} \int_{\Gamma} G_{\kappa}(\mathbf{x}, \mathbf{y}) \boldsymbol{\lambda}_{h}(\mathbf{x}) \boldsymbol{\mu}_{h}(\mathbf{y}) \mathrm{d} S(\mathbf{x}) \mathrm{d} S(\mathbf{y})
$$

for piecewise linear tangential vector fields, and

$$
\begin{aligned}
\left(\tilde{\mathrm{B}} \lambda_{h}, \mathbf{v}_{h}\right)_{\boldsymbol{\tau}}=\int_{\Gamma} \int_{\Gamma} & \left(\lambda _ { h } ( \mathbf { y } ) \left(\mathbf{n}(\mathbf{x}) \cdot \operatorname{grad}_{\mathbf{x}} G_{\kappa}(\mathbf{x}, \mathbf{y})\right.\right. \\
& \left.\left.-\operatorname{grad}_{\mathbf{x}} G_{\kappa}(\mathbf{x}, \mathbf{y})\left(\lambda_{h}(\mathbf{y}) \cdot \mathbf{n}(\mathbf{x})\right)\right)\right) \mathbf{v}_{h}(\mathbf{x}) \mathrm{d} S(\mathbf{x}) \mathrm{d} S(\mathbf{y})
\end{aligned}
$$


for piecewise constant $\varphi$ and piecewise linear $\mathbf{v}_{h}$. For the case $\kappa=0$ the singular inner integral can be evaluated analytically, whereas low-order Gauss quadrature can be applied to the outer integrals [34, Section 4.3]. For general $\kappa$ we remark that, for instance, $A^{\kappa}-A^{0}$ is an integral operator with an analytic kernel. Hence, low-order quadrature will also yield sufficient accuracy for the inner integral.

In order to resolve complicated geometries and to achieve sufficient accuracy, fine surface meshes have to be used. More than $10^{4}$ elements are not unusual. However, when (36) is written as a system of linear equations, most matrices are dense. As a consequence, memory requirements for merely storing the matrices will already exceed several GBytes. Therefore, a compression technique must be applied to the different discrete boundary-integral operators. This can be done by using the $\mathcal{H}^{2}$-matrix approximation [35]. The idea is to separate nearfield and far-field interactions: conventional evaluation is used for the near field, whereas multidimensional Chebychev interpolation is used to approximate the smooth kernel in the far field by tensor-product polynomials. This amounts to an approximation of the discrete integral operator that can give sufficient accuracy, while dramatically reducing memory usage and speeding up the evaluation of the discrete boundary-integral operators. Details and numerical experiments can be found in [30]. We remark that there is a close relationship of the $\mathcal{H}^{2}$-matrix technique and the fast multipole method for integral operators [36].

The compressed operators only permit us to evaluate their products with coefficient vector. Hence, the use of iterative solvers for (36) is mandatory. By separating real and imaginary parts of the unknowns, we can convert (36) into a symmetric system of linear equations, which can be solved by the conjugate residual method [37, Section 9.5]. Yet, since we tackle integral equations of the first kind, the conditioning of the discrete system (36) will deteriorate on fine meshes: the iterative solver will stall unless a good preconditioner is supplied. It turns out that using the diagonal of the system (36) is a good choice, see [15, Section 9]. Numerical investigations are reported in [34, Section 5.2].

\section{Postprocessing}

As we remarked in the introduction, getting approximate Cauchy data $\left(\mathbf{u}_{h}, \lambda_{h}\right)$ might not be the eventual goal of the computation. Thus, we have to figure out how to get (i) the total Ohmic losses in $\Omega_{C}$, and (ii) the total force acting on $\Omega_{C}$.

Ohmic losses are the only mechanism for the dissipation of field energy in the eddy-current model. Moreover, since all fields are harmonic in time, the total field energy inside $\Omega_{C}$ will not change over one period. Therefore, we get the averaged Ohmic losses by appealing to Poynting's theorem

$$
\bar{P}_{\mathrm{Ohm}}=-\frac{1}{2} \mathfrak{R e}\left\{\int_{\Gamma}(\mathbf{E} \times \overline{\mathbf{H}}) \cdot \mathbf{n} \mathrm{d} S\right\}=\frac{1}{2} \omega \mathfrak{I m}\left\{(\mathbf{u}, \lambda)_{\tau}\right\},
$$

where we used $\mathbf{u}=\gamma_{D}^{-} \mathbf{A}=(i \omega)^{-1} \gamma_{D}^{-} \mathbf{E}$ and $\lambda=1 / \mu_{c} \gamma_{N}^{-} \mathbf{A}=\mathbf{H} \times \mathbf{n}$. Therefore, we will approximate

$$
\bar{P}_{\mathrm{Ohm}} \approx \bar{P}_{\mathrm{Ohm}}^{h}:=\frac{1}{2} \omega \mathfrak{I m}\left(\mathbf{u}_{h}, \lambda_{h}\right)_{\boldsymbol{\tau}} .
$$

The error can be estimated by

$$
\begin{aligned}
\bar{P}_{\mathrm{Ohm}}-\bar{P}_{\mathrm{Ohm}}^{h} & =\frac{1}{2} \omega \mathfrak{I m}\left\{\left(\mathbf{u}, \boldsymbol{\lambda}-\boldsymbol{\lambda}_{h}\right)_{\boldsymbol{\tau}}+\left(\mathbf{u}-\mathbf{u}_{h}, \boldsymbol{\lambda}_{h}\right)_{\boldsymbol{\tau}}\right\} \\
& \leq \frac{1}{2} \omega\left(\|\mathbf{u}\|_{\mathrm{el}}\left\|\boldsymbol{\lambda}-\boldsymbol{\lambda}_{h}\right\|_{\mathrm{mag}}+\left\|\mathbf{u}-\mathbf{u}_{h}\right\|_{\mathrm{el}}\left\|\boldsymbol{\lambda}_{h}\right\|_{\mathrm{mag}}\right),
\end{aligned}
$$


where we have exploited the continuity of the pairing $(\cdot, \cdot)_{\tau}$. This shows that $\bar{P}_{\mathrm{Ohm}}^{h}$ will converge at the same rate as observed for the Cauchy data.

To compute the total force on the conductor, we can resort to the magnetic Maxwell stress tensor for linear materials [38, Section 6.7]

$$
\mathbb{T}:=\mathcal{B} \cdot \mathcal{H}^{T}-\frac{1}{2}(\mathcal{B} \cdot \mathcal{H}) \mathbb{I},
$$

where, $\mathcal{B}$ and $\mathcal{H}$ denote the real, time-dependent fields. Ignoring the electric forces is consistent with the eddy-current model, which rests on the assumption of negligible electric-field energy. Next, we consider $\mathbb{T}$ on $\Gamma$ and split both the magnetic induction $\mathcal{B}$ and $\mathcal{H}$ into tangential and normal components, $c f$. [39, Section 6].

$$
\mathcal{B}(\mathbf{x})=\mathcal{B}_{n}(\mathbf{x}) \mathbf{n}(\mathbf{x})+\mathcal{B}_{\mathbf{t}}(\mathbf{x}), \quad \mathcal{H}(\mathbf{x})=\mathcal{H}_{n}(\mathbf{x}) \mathbf{n}(\mathbf{x})+\mathcal{H}_{\mathbf{t}}(\mathbf{x}), \quad \mathbf{x} \in \Gamma .
$$

Using the constitutive equation $\mathcal{B}=\mu_{0} \mathcal{H}$, that is valid in $\Omega^{\prime}$, we express

$$
\mathcal{H}_{n}(\mathbf{x})=\frac{1}{\mu_{0}} \mathcal{B}_{n}, \quad \mathcal{B}_{\mathbf{t}}(\mathbf{x})=\mu_{0} \mathcal{H}_{\mathbf{t}}
$$

and get on $\Gamma$

$$
\mathbb{T}(\mathbf{x}) \mathbf{n}(\mathbf{x})=\frac{1}{2}\left(\frac{1}{\mu_{0}} \mathcal{B}_{n}^{2}(\mathbf{x})-\mu_{0}\left|\mathcal{H}_{\mathbf{t}}\right|^{2}\right) \mathbf{n}(\mathbf{x})+\mathcal{B}_{n}(\mathbf{x}) \mathcal{H}_{\mathbf{t}}(\mathbf{x}), \quad \mathbf{x} \in \Gamma .
$$

Hence, the total force on the conductor at a particular time is given by

$$
\begin{aligned}
F_{\text {tot }} & =\int_{\gamma} \mathbb{T}(\mathbf{y}) \mathbf{n}(\mathbf{y}) \mathrm{d} S(\mathbf{y}) \\
& =\int_{\gamma} \frac{1}{2}\left(\frac{1}{\mu_{0}} \mathcal{B}_{n}^{2}(\mathbf{y})-\mu_{0}\left|\mathcal{H}_{\mathbf{t}}(\mathbf{y})\right|^{2}\right) \mathbf{n}(\mathbf{y})+\mathcal{B}_{n}(\mathbf{y}) \mathcal{H}_{\mathbf{t}}(\mathbf{y}) \mathrm{d} S(\mathbf{y}) .
\end{aligned}
$$

Let us revert to complex amplitudes $\mathbf{B}$ and $\mathbf{H}$, for which the averaged force over one period is given by

$$
\bar{F}_{\text {tot }}=\int_{\gamma} \frac{1}{4}\left(\frac{1}{\mu_{0}}\left|B_{n}(\mathbf{y})\right|^{2}-\mu_{0}\left|\mathbf{H}_{\mathbf{t}}(\mathbf{y})\right|^{2}\right) \mathbf{n}(\mathbf{y})+\frac{1}{2} \mathfrak{R e}\left\{B_{n}(\mathbf{y}) \mathbf{H}_{\mathbf{t}}(\mathbf{y})\right\} \mathrm{d} S(\mathbf{y}) .
$$

From $\mathbf{B}=$ curlA we infer $B_{n}=\operatorname{curl}_{\Gamma} \mathbf{u}$, where $\operatorname{curl}_{\Gamma}$ stands for the scalar surface rotation $\left(\operatorname{div}_{\Gamma}\right.$ applied to the rotated field). On the other hand, it is straightforward that $\mathbf{H}_{\mathbf{t}}=-\boldsymbol{\lambda} \times \mathbf{n}$. Thus, we can rewrite

$$
\begin{aligned}
\bar{F}_{\text {tot }}=\int_{\gamma} & \left(\frac{1}{4 \mu_{0}}\left|\operatorname{curl}_{\Gamma} \mathbf{u}(\mathbf{y})\right|^{2}-\frac{\mu_{0}}{4}|\lambda(\mathbf{y})|^{2}\right) \mathbf{n}(\mathbf{y}) \\
& -\frac{1}{2} \mathfrak{R e}\left\{\operatorname{curl}_{\Gamma} \mathbf{u}(\mathbf{y})(\lambda(\mathbf{y}) \times \mathbf{n}(\mathbf{y}))\right\} \mathrm{d} S(\mathbf{y}) .
\end{aligned}
$$

Finally, we have expressed the total force in terms of quantities that occur as unknowns in the variational problem (28). Now, it is straightforward how to compute an approximation of $\bar{F}_{\text {tot }}$ from the boundary-element solution $\left(\mathbf{u}_{h}, \lambda_{h}\right)$. As far as the approximation error is concerned, the same considerations apply as for the energy flux.

It is important to be aware that the force as given by (38) is by no means a continuous functional in the natural trace norms, because the inclusions $\mathbf{X}_{\mathrm{el}}(\Gamma) \subset \boldsymbol{L}^{2}(\Gamma)$ and $\mathbf{X}_{\mathrm{mag}}(\Gamma) \subset$ $\boldsymbol{L}^{2}(\Gamma)$ do not hold (Compare the case to the Neumann trace space $H^{-1 / 2}(\Gamma)$ for second-order elliptic problems). Of course, Equation (38) can easily be evaluated for the boundary-element functions, but unlike in the case of the total energy flux, rates of convergence for $\bar{F}_{\text {tot }}$ cannot be inferred from (33). 
Remark 7.1. Formally, the expression under the integral in (38) is a surface-force density, although this physical interpretation is dubious. However, one should remember the skin effect that leads to an exponential decay of electromagnetic fields penetrating conductors. If $(\omega \sigma \mu)^{-1 / 2}$ is small compared to the size of the conductor, an argument using a Gaussian "pillbox" will show that the integrand can be viewed as a reasonable approximation of a surface force density.

Remark 7.2. We emphasize that approximations for the traces of the fields onto $\Gamma$ are directly available, because we have relied on a direct boundary element method. If an indirect method had been used, it would have taken expensive post-processing, in order to get the same information.

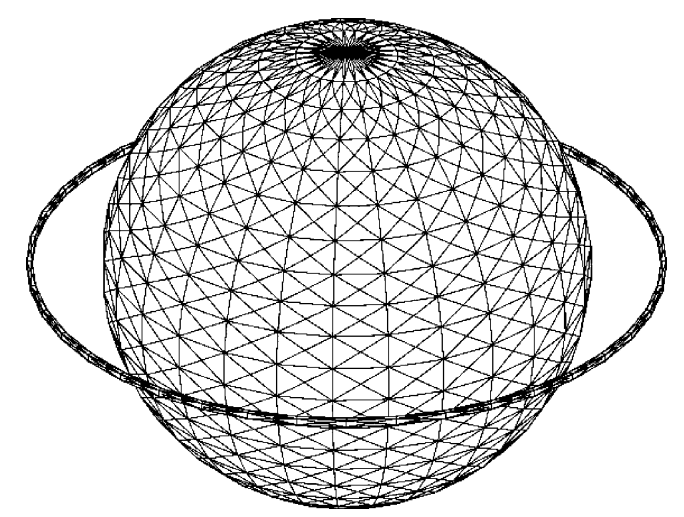

Figure 3. Surface mesh $\Gamma_{h}$ for the sphere and inductor loop.

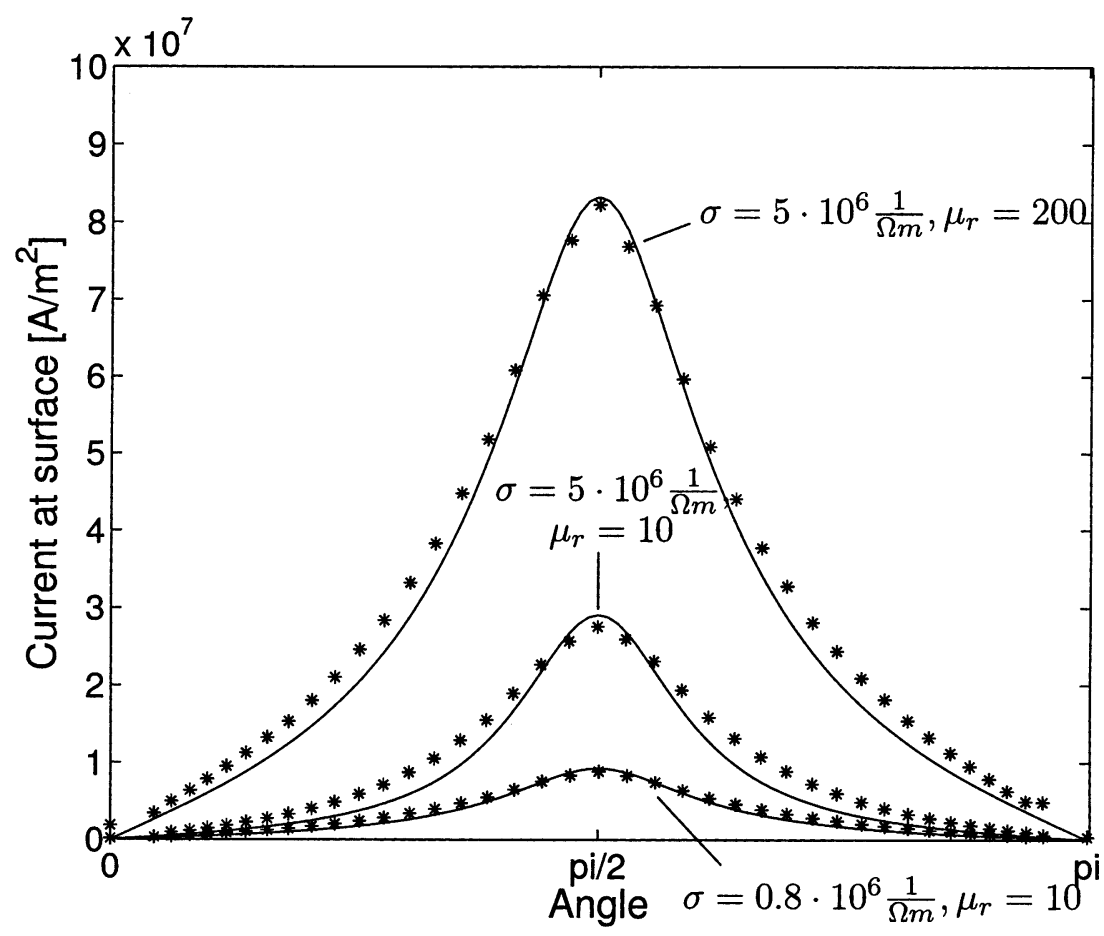

Figure 4. Comparison of analytical solution for equivalent surface current and solution obtained by impedance model and symmetrically coupled boundary-element scheme. The current density along a meridian is plotted. 


\section{Numerical results}

In a numerical experiment, the conductor $\Omega_{C}$ is a homogeneous sphere with radius $5 \mathrm{~cm}$. A surface impedance condition according to (12) is used. An exiting current of $1 \mathrm{kA}$ with frequency $\omega=2 \pi \times 10 \mathrm{kHz}$ is imposed in a thin loop circling the equator of the sphere. This arrangement has been chosen, because an analytic solution of the eddy-current problem is available. The surface mesh $\Gamma_{h}$ is displayed in Figure 3. It comprises 2214 faces, 3321 edges, and 1109 vertices. Thus, a system of equations with 4430 complex unknowns has to be solved.

In Figure 4 the modulus of the equivalent surface current $\mathbf{H} \times \mathbf{n}$ and its approximation $\lambda_{h}$ are plotted along a meridian of the sphere and for different material parameters $\sigma$ and $\mu_{r}$ inside $\Omega_{C}$. A good agreement of analytical and numerical solution can be observed, which is hardly surprising, as for a smooth object like the sphere impedance boundary conditions work well and a relatively coarse surface mesh already yields good approximation.

\section{Conclusion}

We have presented a direct boundary-element method for the time-harmonic, linear eddy-current problem. This approach merely relies on a mesh on the surface of the conductor and is not affected by boundary layers due to the skin effect. Its unknowns are related to approximations of traces of physical fields on the surface of the conductor and for those it supplies quasi-optimal Galerkin boundary-element solutions. Approximate total Ohmic losses and forces can easily be recovered from the approximate surface traces.

A significant improvement of the method can be achieved by using a priori anisotropically graded meshes adapted to the singular behaviour of the fields at edges and corners of the conductors, $c f$. [40,41]. These field singularities are now well understood [42]. The authors are convinced that combining the boundary-element method described in the article with a priori adaptivity will yield a very efficient numerical method that will outperform approaches based on discretizing three-dimensional volumes.

\section{References}

1. Arnulf Kost, Numerische Methoden in der Berechnung elektromagnetischer Felder. Berlin: Springer (1994) $405 \mathrm{pp}$.

2. D. Colton and R. Kress, Integral Equation Methods in Scattering Theory. Pure and Applied Mathematics. New York: John Wiley \& Sons (1983) 271 pp.

3. J.-C. Nédélec, Acoustic and Electromagnetic Equations: Integral Representations for Harmonic Problems. Volume 44 of Applied Mathematical Sciences. Berlin: Springer-Verlag (2001) 316 pp.

4. D.R. Wilton, Review of current status and trends in the use of integral equations in computational electromagnetics. Electromagnetics 12 (1992) 287-341.

5. G.C. Hsiao, Mathematical foundations for the boundary field equation methods in acoustic and electromagnetic scattering. In: F. Santosa and I. Stakgold (eds.), Analysis and Computational Methods in Scattering and Applied Mathematics. A Volume in the Memory of Ralph Ellis Kleinman. Volume 147 of Research Notes in Mathematics. Boca Raton, FL: Chapman \& Hall, CRC (2000) pp. 149-163.

6. I.D. Mayergoyz, 3D eddy current problems and the boundary integral equation method. In: Z.J. Cendes (ed), Computational Electromagnetics. Amsterdam: Elsevier (1986) pp. 163-171.

7. J. Shen, Computational Electromagnetics Using Boundary Elements. Volume 24 of Topics in Engineering. Southampton, Boston: Computational Mechanics Publ (1995) $264 \mathrm{pp}$

8. D. Zheng, Three-dimensional eddy current analysis by the boundary element method. IEEE Trans. Magn. 33 (1997) 1354-1357.

9. J. Yuan, X. Ma and X. Cui, Three-dimensional eddy current calculation by an adaptive three-component boundary element algorithm. IEEE Trans. Magn. 33 (1997) 1275-1278. 
10. K. Ishibashi, Eddy current analysis by integral equation method utilizing loop electric and surface magnetic currents as unknowns. IEEE Trans. Magn. 34 (1998) 2585-2588.

11. S. Kurz, J. Fetzer, G. Lehner and W.M. Rucker, A novel formulation for 3D eddy current problems with moving bodies using a Lagrangian description and BEM-FEM coupling. IEEE Trans. Magn. 34 (1998) 3068-3073.

12. M. Costabel, Symmetric methods for the coupling of finite elements and boundary elements. In: C.A Brebbia and W.L. Wendland and G. Kuhn (eds.), Boundary Elements IX Berlin: Springer-Verlag (1987) pp. $411-420$.

13. A. Bossavit, A rationale for edge elements in 3D field computations. IEEE Trans. Magn. 24 (1988) 74-79.

14. J.C. Nédélec, Mixed finite elements in $\mathbb{R}^{3}$. Numer. Math. 35 (1980) 315-341.

15. R. Hiptmair, Symmetric coupling for eddy current problems. SIAM J. Numer. Anal. 40 (2002) 41-65.

16. H.K. Dirks, Quasi-stationary fields for microelectronic applications. Electr. Eng. 79 (1996) 145-155.

17. H. Ammari, A. Buffa and J.-C. Nédélec, A justification of eddy currents model for the Maxwell equations. SIAM J. Appl. Math. 60 (2000) 1805-1823.

18. A. Bossavit, Computational Electromagnetism. Variational Formulation, Complementarity, Edge Elements. Volume 2 of Electromagnetism Series. San Diego, CA: Academic Press (1998) 352 pp.

19. A. Alonso-Rodriguez, P. Fernandes and A. Valli, Weak and strong formulations for the time-harmonic eddycurrent problem in general multi-connected domains. Eur. J. Appl. Math. 14 (2003) 387-406.

20. W. McLean, Strongly Elliptic Systems and Boundary Integral Equations. Cambridge, UK: Cambridge University Press (2000) $357 \mathrm{pp}$.

21. R.C. McCamy and E.P. Stephan, Solution procedures for three-dimensional eddy-current problems. J. Math. Anal. Appl. 101 (1984) 348-379.

22. D. Colton and R. Kress, Inverse Acoustic and Electromagnetic Scattering Theory. Volume 93 of Applied Mathematical Sciences, 2nd edition. Heidelberg: Springer (1998) 334 pp.

23. A. Buffa, Traces theorems on non-smmoth boundaries for functional spaces related to Maxwell equations: An overwiew. In: C. Carstensen and S. Funken, W. Hackbusch, R.H.W. Hoppe and P. Monk (eds.), Computational Electromagnetics, Volume 28 of Lecture Notes in Computational Science and Engineering, Berlin: Springer (2003), pp. 23-34.

24. A. Buffa, M. Costabel and D. Sheen, On traces for $\mathbf{H}(\mathbf{c u r l}, \Omega)$ in Lipschitz domains. J. Math. Anal. Appl. 276 (2002) 845-867.

25. A. Buffa, R. Hiptmair, T. von Petersdorff and C. Schwab, Boundary element methods for Maxwell equations on Lipschitz domains. Numer. Math. 95 (2003) 459-485 Published online (DOI 10.1007/s00211-002-0407-z).

26. A. Buffa, M. Costabel and C. Schwab, Boundary element methods for Maxwell's equations on non-smooth domains. Numer. Math. 92 (2002) 679-710.

27. M. Teltscher, E.P. Stephan and M. Maischak, A residual error estimator for an electromagnetic FEM-BEM coupling problem in $\mathbb{R}^{3}$. Report, Institut für Angewandte Mathematik, Universität Hannover, Hannover, Germany (2003) 31 pp.

28. R. Hiptmair, Finite elements in computational electromagnetism. Acta Numerica 11 (2002) 237-339.

29. F. Brezzi and M. Fortin, Mixed and Hybrid Finite Element Methods. Berlin: Springer (1991) 350 pp.

30. S. Börm and J. Ostrowski, Fast evaluation of boundary integral operators arising from an eddy current problem. J. Comp. Phys. 193 (2003) 67-85.

31. P.G. Ciarlet, The Finite Element Method for Elliptic Problems. Volume 4 of Studies in Mathematics and its Applications. Amsterdam: North-Holland (1978) 580 pp.

32. A. Buffa and S.A. Christiansen, The electric field integral equation on Lipschitz screens: Definition and numerical approximation. Numer. Math. 94 (2002) 229-267.

33. R. Hiptmair and J. Ostrowski, Generators of $H_{1}\left(\Gamma_{h}, \mathbb{Z}\right)$ for triangulated surfaces: Construction and classification. SIAM J. Computing 31 (2002) 1405-1423.

34. J. Ostrowski, Boundary Element Methods for Inductive Hardening. Fakultät für Mathematik und Physik, Tübingen, November (2002) $123 \mathrm{pp}$.

35. S. Börm and W. Hackbusch, $\mathcal{H}^{2}$-matrix approximation of integral operators by interpolation. Appl. Num. Math. 43 (2002) 129-143.

36. V. Rokhlin, Rapid solution of integral equations of scattering theory in two dimensions. J. Comput. Phys. 86 (1990) 414-439.

37. W. Hackbusch, Iterative Solution of Large Sparse Systems of Equations. Volume 95 of Applied Mathematical Sciences. New York: Springer-Verlag (1993) 429 pp.

38. J.D. Jackson, Classical Electrodynamics. 2nd edition. New York: Wiley (1975) 808 pp. 


\section{$250 \quad$ R. Hiptmair and J. Ostrowski}

39. S. Kurz, J. Fetzer, G. Lehner and W.M. Rucker, Numerical analysis of three-dimensional eddy current problems with moving bodies by boundary element-finite element coupling, Surv. Math. Ind. 9 (1999) 131-150.

40. A. Buffa and M. Costabel and M. Dauge, Algebraic convergence for anisotropic edge elements in polyhedral domains. Technical Report 28-PV, IMATI-CNR, Pavia, Italy (2003) 34 pp.

41. S. Nicaise, Edge elements on anisotropic meshes and approximation of the Maxwell equations. SIAM $J$. Numer. Anal. 39 (2001) 784-816.

42. M. Costabel, M. Dauge and S. Nicaise, Singularities of eddy current problems. ESAIM: Math. Model. Numer. Anal. 37, (2003) 807-831. 